\title{
Conserved Intramolecular Interactions Maintain Myosin Interacting-Heads Motifs Explaining Tarantula Muscle Super-Relaxed State Structural Basis
}

\author{
Lorenzo Alamo ${ }^{1}$, Dan $\mathrm{Qi}^{2}$, Willy Wriggers ${ }^{3}$, Antonio Pinto ${ }^{1}$, Jingui Zhu ${ }^{2}$, Aivett Bilbao ${ }^{1 \mathrm{a}}$, Richard \\ E. Gillilan ${ }^{4}$, Songnian $\mathrm{Hu}^{2}$ and Raúl Padrón ${ }^{1, *}$ \\ ${ }^{1}$ Centro de Biología Estructural, Instituto Venezolano de Investigaciones Científicas (IVIC), Apdo. \\ 20632, Caracas 1020A, Venezuela. ${ }^{2}$ Key Laboratory of Genome Sciences and Information, Beijing \\ Institute of Genomics, China. ${ }^{3}$ Department of Mechanical and Aerospace Engineering, Old \\ Dominion University, Norfolk, Virginia 23529, U.S.A. ${ }^{4}$ Macromolecular Diffraction Facility, \\ Cornell High Energy Synchrotron Source, Ithaca, New York, U.S.A.
}

Present address: ${ }^{a}$ SIB Swiss Institute of Bioinformatics, Proteome Informatics Group and Life Sciences Mass Spectrometry, School of Pharmaceutical Sciences, University of Geneva, Geneva, Switzerland

*Corresponding author. E-mail: raul.padron@gmail.com, Tel +58 212504 1098, Fax +58 212504 1444

E-mail addresses: Lorenzo Alamo: lorenzo.alamo@gmail.com, Dan Qi: isaacruby@gmail.com, Willy Wriggers: wwrigger@odu.edu, Antonio Pinto: pinto.misle@gmail.com, Jingui Zhu: osmanthusz@gmail.com, Aivett Bilbao: aivettbilbao@gmail.com, Richard Gillilan: reg8@ cornell.edu, Songnian Hu: husn@big.ac.cn, Raúl Padrón: raul.padron@gmail.com

\begin{abstract}
Tarantula striated muscle is an outstanding system for understanding the molecular organization of myosin filaments. 3D reconstruction based on cryo-EM images and single-particle image processing revealed that in a relaxed state, myosin molecules undergo intramolecular head-head interactions, explaining why head activity switches off. The filament model obtained by rigidly docking a chicken smooth muscle myosin structure to the reconstruction was improved by flexibly fitting an atomic model built by mixing structures from different species to a tilt-corrected 2-nm 3D map of frozen-hydrated tarantula thick filament. We used heavy and light chain sequences from tarantula myosin to build a single-species homology model of two heavy meromyosin interactingheads motifs (IHMs). The flexibly fitted model includes previously missing loops and shows five intramolecular and five intermolecular interactions that keep the IHM in a compact off structure, forming four helical tracks of IHMs around the backbone. The residues involved in these interactions are oppositely charged, and their sequence conservation suggests that IHM is present across animal species. The new model, PDB 3JBH, explains the structural origin of the ATP turnover rates detected in relaxed tarantula muscle by ascribing the very slow rate to docked unphosphorylated heads, the slow rate to phosphorylated docked heads, and the fast rate to phosphorylated undocked heads. The conservation of intramolecular interactions across animal species and presence of IHM in bilaterians suggest that a super-relaxed state should be maintained, as it plays a role in saving ATP in skeletal, cardiac, and smooth muscle.
\end{abstract}

Keywords: Myosin thick filament, myosin interacting-heads motif, cryo-electron microscopy, striated muscle, super-relaxation.

Abbreviations used: BH: blocked head; C loop: cardiac loop; CM loop: cardiomyopathy loop; ELC: essential light chain; FH: free head; HMM: heavy meromyosin; IHM: interacting-heads motif; MHC: myosin II heavy chain; NTE: N-terminal extension; RLC: regulatory light chain; S2: subfragment 2; SAXS: small angle X-ray solution scattering; SRX: super-relaxed state

Running title: Relaxed myosin interacting-heads motif structure 


\section{Introduction}

Muscle contraction involves the interaction of two sets of filaments: actin-containing thin filaments and myosin-containing thick filaments. Muscle relaxation is an important step of contraction, and thick filaments are essential for relaxation. ${ }^{1}$ The two myosin II coiled-coil heavy chains (MHCs) form a tail with two heads, with attached regulatory light chains (RLCs) and essential light chains (ELCs) on each. The tails pack together, comprising the thick filament backbone with protruding heads that form helical tracks. 3D reconstructions have shown that the number of helical tracks in striated muscle varies between three in cardiac vertebrates (mouse, ${ }^{2}$ human, ${ }^{3}$ zebrafish ${ }^{4}$ ), four in arthropods (tarantula, ${ }^{5}$ Limulus, $^{6}$ scorpion $^{7}$ ), and seven in mollusks (scallop). ${ }^{8}$ The number of smooth muscle helical tracks is four in Platyhelminthes (Schistosome) ${ }^{9}$ in vertebrates, smooth muscle filaments are non-helical and side-polar. ${ }^{10}$

Electron microscopy studies of smooth muscle myosin molecules suggest that an off state is achieved by asymmetric, intramolecular interaction between the actin-binding region of one myosin head and the converter region of the other, which switches off both heads. ${ }^{11}$ This mechanism of relaxation, the so-called myosin interacting-heads motif (IHM), ${ }^{5}$ was shown to underlie the relaxed state of thick filaments from striated muscle. ${ }^{5}$ The IHM is present in species separated by at least 600 million years of independent evolution. It has been observed by electron microscopy in thick filaments from the striated muscle of arthropods, ${ }^{5-7}$ mollusks ${ }^{8}$, the cardiac muscle of vertebrates, ${ }^{2-4}$ and the smooth muscle of Platyhelminthes. ${ }^{12}$ Electron microscopy has also shown that the motif is present in isolated myosin molecules of intrinsically regulated molecules (like tarantula and Limulus striated muscle and non-muscle myosin IIA) and in unregulated myosins (like skeletal and cardiac muscle). ${ }^{13}$ Recently, the motif has been detected on isolated myosin molecules from the smooth muscle of the Cnidarians' giant sea anemone (Condylactis gigantea) ${ }^{14}$ but not in isolated myosins from the amoeba Acanthamoeba castellani. ${ }^{14}$ Finally, the presence of the IHM in squid (Loligo peali) has been inferred by comparing the small angle X-ray solution scattering (SAXS) profile of its striated muscle with the predicted scattering profile for tarantula Protein Data Bank (PDB) 3DTP structure. $^{15}$

The uniqueness, wide presence, and high conservation of the IHM lead to its fundamental functional importance in nature as the simplest conserved structural mechanism that explains the relaxed (off) state in muscle. The IHM is established only in relaxing conditions. In the presence of Mg.ATP, heads are bent at the "pliant region," 16 as the switch 2 element is closed, inhibiting the phosphate release. The bending of free heads and blocked heads produce the required conformations for establishing several head-head and head-tail intramolecular interactions as well as several head-head and head-backbone intermolecular interactions, which allow the formation of thick filaments in regulated muscles. It has been suggested that intramolecular interactions are a general mechanism for inducing muscle relaxation and switching off myosin II-based motile activity in both muscle and non-muscle cells. ${ }^{17}$ The so called super-relaxed (SRX) state of myosin II has been reported in striated muscle and is characterized by a sub-population of myosin heads with a highly inhibited rate of ATP turnover, lower than the turnover observed for single molecules. ${ }^{18}$ Myosin IHM has been found to be involved in the slow ATP turnover rate observed in vertebrate skeletal and cardiac muscle fibers. ${ }^{19-21}$ In particular, relaxed tarantula striated muscle fibers exhibit a very slow rate. ${ }^{22}$

The tarantula striated muscle IHM model (PDB 3DTP) has improved our understanding of the activation mechanism in arthropods that involves two phosphorylatable serines (Ser35 and Ser45). ${ }^{23}$ Based on this structure, a cooperative phosphorylation activation (CPA) mechanism (Fig. 8C-F) as well as a model for activation, potentiation, and post-tetanic potentiation involving swaying heads in a relaxed state were proposed for tarantula striated muscle. ${ }^{24,}{ }^{25} \mathrm{~A}$ disorder-to-order molecular mechanism occurring in the myosin RLC N-terminal extension (NTE) is proposed to control this 
arthropod phosphorylation-based activation. ${ }^{26,27}$ The IHM model has also improved knowledge of the activation mechanism in vertebrate skeletal ${ }^{28,29}$ and cardiac muscle. ${ }^{30,31}$ On the other hand, the smooth muscle IHM (PDB 1I84) structure has improved knowledge of its activation mechanism, ${ }^{32}$ including a possible role of myosin ELC. ${ }^{33}$

An enhanced model of the IHM is very important for increasing our understanding of the thick filament relaxation and activation mechanisms. The only structural information for the IHM comes from cryo-EM of chicken smooth muscle myosin II $2 \mathrm{D}$ crystals ${ }^{11,34,35}$ and from tarantula striated muscle frozen-hydrated relaxed thick filaments. ${ }^{5,23}$ The quasi-atomic model smooth muscle IHM (PDB 1I84) includes the chicken smooth muscle subfragment 1 atomic structure (PDB 1BR1), but as it did not come from a filament, it does not properly include a subfragment 2 (S2) crystal structure. ${ }^{11}$ Two reconstructions were calculated from low-dose electron micrographs of frozenhydrated tarantula thick filaments. The first reconstruction reached a resolution of $2.5 \mathrm{~nm}^{5}$ and was used to build an initial quasi-atomic model of the filament by manually fitting the chicken smooth muscle myosin quasi-atomic structure PDB $1 \mathrm{I} 84^{11}$ without the S2. The second reconstruction, which was deposited in the Electron Microscopy Data Bank (EMDB) ${ }^{36}$ as EMDB-1950, ${ }^{23}$ reached a slightly higher resolution $(2.0 \mathrm{~nm})$ by using a higher number of image segments and by including the tilt angle made by the filaments with the grid in the reconstruction. This second reconstruction was more detailed, clearly showing two new interactions in addition to the five shown in the first reconstruction. ${ }^{5}$ This enhanced reconstruction led to a second improved tarantula IHM model (PDB 3DTP), ${ }^{23}$ which was achieved by flexible fitting of an atomic model built from different species: a human cardiac myosin S2 crystal structure (PDB 2FXM), ${ }^{37}$ the predicted secondary structure homologous quasi-atomic model for the tarantula (Avicularia) myosin RLC sequence, ${ }^{23}$ and the ELC and myosin heads' motor domain from the chicken smooth muscle quasi-atomic model (PDB 1I84). ${ }^{35}$ Since three loops (loop 1, loop 2, and loop NATP-V, which is defined here as the loop near the nucleotide ATP binding D458 shown in Fig. 6b) are missing in chicken myosin II heavy chain (MHC) (PDB 1BR1), their sequences are not present in two earlier IHM PDB models (PDB 1I84, PDB 3DTP).

Analysis of transcriptional isoforms of tarantula skeletal muscle protein sequences has determined its myosin ELC and RLC sequences. ${ }^{38}$ The aims of the present work are: (1) to build a homologous IHM quasi-atomic model (PDB 3JBH) based on PDB 3DTP using the tarantula myosin II ELC, ${ }^{38}$ RLC, ${ }^{38}$ and MHC sequences reported here to flexibly fit this model to the tarantula thick filament 3D reconstruction (EMD-1950); (2) to analyze whether the residues involved in maintaining the critical intramolecular interactions of the IHM could establish ionic interactions and whether they are conserved across different species; and (3) to investigate whether this new homologous tarantula IHM model and its interactions provide clues about the structural basis of the SRX state in tarantula striated muscle.

\section{Results}

\section{Tarantula myosin MHC, ELC and RLC residue sequences:}

The tarantula Aphonopelma sp. MHC sequence $\left(\mathrm{GenBank}^{39}\right.$ KT619079) was determined as described in Materials and Methods, which is included in the supplementary data (see Accession numbers) along with the myosin ELC $^{38}$ (GenBank KT390185) and RLC ${ }^{38}$ (GenBank KT390186). The tarantula Avicularia avicularia myosin RLC sequence (UNIPROT ${ }^{40} \underline{B} 4 X T 43^{23}$ that was included in PDB 3DTP is very similar to that of Aphonopelma sp., as both have 196 amino acids and differ only on 9 .

\section{Tarantula IHM quasi-atomic model:}


We built a homologous single-species model using the MHC, RLC, and ELC of tarantula Aphonopelma sp. based on PDB 3DTP ${ }^{23}$ (see Materials and Methods). The model was flexibly fit into the 2-nm resolution 3D map (Fig. 1, EMD-1950) calculated from electron micrographs of rapidly frozen tarantula Aphonopelma sp. relaxed thick filaments preserved in vivo in a relaxed state. ${ }^{23}$ The model includes three modeled loops (loop 1, loop 2, loop NATP-V; Suppl. Table 1) that are missing in the chicken MHC crystal structure (PDB 1BR1) and the PDB 1I84 and PDB 3DTP models. Due to the IHM structural asymmetry of free and blocked heads, the loops are located in very different environments (Fig. 2a). Loop 1 faces the solvent in both the free and blocked head (Fig. 2a) and is not involved in any intra- or intermolecular interactions. Loop 2 was included after being remodeled de novo (Fig. 2a, see Materials and Methods) and is fitted to density "a" (Fig. 2b). The loop 2 sequence in myosin II significantly affects actin-activated ATPase activity, mechanochemical coupling, and actin binding. Regulation by RLC phosphorylation depends on the presence of a loop 2 with greater length and a less positive charge density than the skeletal isoform. ${ }^{41-43}$ The tarantula loop 2 sequence appears to be different from other species but preserves the positively charged region, a so-called lysine pocket (Suppl. Figs. 1, 2). ${ }^{42}$ The CM loop, previously fitted to density "a," ${ }^{23,37}$ was found after remodeling the missing loops to better match the densities in the interface with the next IHM (Fig. 2a, Suppl. Movies 2-4) and loop 2 to better match interaction "a." The flexible fitting of the free head cluster of loops (Fig. 2a, top right) to the 3D map required some rearrangement of the myosin subfragment $150 \mathrm{~K}$ domain, including some cleft closure between the upper and lower parts of the $50 \mathrm{~K}$ domain. It was previously proposed that the apparent cleft closure and specific CM loop orientation were required to guide the free head, which was detached after the power stroke, to establish the precise electrostatic docking interaction "a" onto ring 2 of $S 2$, a requisite for reforming the helical tracks of IHMs. ${ }^{23}$ This remodeling suggests that it is loop 2, not the CM loop, that is responsible for this electrostatic docking interaction.

This quasi-atomic model was deposited as $3 \mathrm{JBH}$ in the Protein Data Bank (PDB). ${ }^{44}$ PDB 3JBH and the earlier PDB 3DTP are very similar (Suppl. Movie 1). In fact, several PDB 3JBH and 3DTP molecule regions are very similar to the crystal structure PDB 1BR1 (Suppl. Movie 1). As these missing loops are located in the region of interaction of one IHM with a neighboring IHM, the flexible fitting process included the densities of this IHM-IHM connecting region (Fig. 2a) located along the IHM helical track (Fig. 1). This fitting approach allows for the inclusion of densities along helical tracks and backbone-anchoring intermolecular interactions, which was not possible with crystals of isolated heavy meromyosin (HMM).

\section{Tarantula model improves upon earlier IHM models:}

The new model, PDB 3JBH, is improved from the PDB 3DTP model since it is built from complete sequences of a single species (tarantula Aphonopelma sp.) rather than a mixture of sequences from three species (human, chicken, and tarantula). In addition to the three new loops, the new model has six surface loops (2, 3, H, 4 or C, CM, and I; Suppl. Table 1) as well as the S2, SH3, catalytic, relay, and converter domains (Fig. 2a, Suppl. Table 1), which are involved in intraand intermolecular interactions (Fig. 2b). The model includes five intramolecular interactions involving the S2 and all six surface loops as well as five intermolecular interactions, two of which are in a cluster of surface loops (C, CM, I, H, and 3), the free and neighboring blocked head regulatory domain, and neighboring myosin tails, and three of which are involved in anchoring with two adjacent myosin tails. The main difference between PDB 3JBH and PDB 3DTP lies mostly in the free head motor domain, where the new, remodeled loops are located near the adjacent blocked head RLC of the next IHM along the helical track (Fig. 1). In addition, PDB 3JBH can be used as a starting model to be flexibly fitted to a higher-resolution 3D reconstruction. ${ }^{45}$

\section{Free and blocked heads of tarantula model have different conformations:}


For myosin heads to form helical tracks in tarantula thick filament, their nucleotide pocket switch 2 elements should be in the "closed" conformation, preventing phosphate release. ${ }^{46,}{ }^{47}$ Also, in the presence of Mg.ATP, the heads should be bent at the "pliant region," closed, inhibiting the phosphate release (Fig. 4). The tarantula IHM model PDB 3JBH offers further insights into the specific conformation of blocked and free head in relaxed filament with a blocked head in a closed pre-power stroke conformation (similar to the MgADP-AlF4, PDB 1BR1) ${ }^{23,} 35$ while the free head, which is also in a pre-power stroke closed conformation (with a shape similar to blocked head at the limited resolution of the 3D map) shows a less angled lever arm than the blocked head (Fig. 4). The angle of the free head lever arm is midway between the lever arms of the blocked head and pre-power stroke transition $1 \mathrm{DFL}^{16}$ (Fig. 4).

\section{Predicted SAXS profile of tarantula model agrees with measured profile of squid HMM:}

X-ray solution scattering of squid HMM in $\mathrm{Ca}^{2+}$-free (EGTA) conditions shows agreement with PDB 3DTP, strengthening the evidence of an ancient IHM compact off state. ${ }^{15}$ In this comparison, the 50 residues of the RLC NTE (which are unique to the tarantula myosin RLC) were deleted. However, the agreement was maintained when they were included (Drs. Jerry H. Brown and Richard Gillilan, personal communication). The computed scattering profile for the tarantula model $(3 \mathrm{JBH})$ closely agrees with the scattering profile computed from PDB 3DTP at angles below $\mathrm{q}=$ $0.11 \AA^{-1}$ (Fig. 5a). While the two computed profiles separate slightly at wider angles, the degree of separation is below the noise level of the current experimentally measured squid HMM profile. ${ }^{15}$ The goodness-of-fit statistic (chi) to the data reported by the software is slightly better for the squid model $(\mathrm{chi}=1.74)$ than for the tarantula model $(\mathrm{chi}=2.01)$, but given the noise levels in the data and the possible systematic deviations of both models from the data at the widest angle, it is not possible to distinguish between the two. Computations extending to a wide angle $(q=1.0 \AA-1$ in Fig. 5b) show that the two models continue to closely agree above $q=0.2 \AA^{-1}$ and are not likely to be distinguished by future SAXS and wide angle X-ray solution scattering experiments in that regime.

\section{Tarantula model includes new intra- and intermolecular interactions:}

In the tarantula thick filament, there were intramolecular interactions (between the domains inside each IHM) ${ }^{5}$ and intermolecular interactions (head-head; between the domains of one IHM and the adjacent IHM along the helical tracks of myosin heads on the surface of the thick filament backbone; Fig. 1, 2) ${ }^{5,23}$ When these interactions are broken, IHMs become less compact as myosin heads are released, ${ }^{24,} 25$ first with the separation and eventual release of free head from its partner blocked head and secondly after the release of blocked head from the thick filament backbone. ${ }^{24} \mathrm{We}$ use the same notation scheme for the five intramolecular interactions ("a," "d," "e," "f," and "g"; Fig. 2b) as suggested previously. ${ }^{23}$ In addition to the three previously described intermolecular interactions s, 23 ("b," "c," and "h"; Fig. 2), we show two new intermolecular interactions (head-tail) between the ELC of the blocked head ("i"; Fig. 3b) and the relay/converter ("j", Fig. 3b) with the neighboring S2. Interactions "h," "i," and "j" cannot be discussed in detail because our myosin tail model does not include this interacting part of the S2 (Fig. 3) and we lack a quasi-atomic model of the subfilament.

\section{Analysis of interactions:}

A rigorous analysis of the residues involved in the intramolecular interactions that keep the tarantula IHM structure compact necessarily requires solving its crystal structure, and a proper analysis of intermolecular interactions requires a resolution of higher than $2 \mathrm{~nm}$ for the 3D map. In the first case, since the tarantula IHM crystal structure had not been determined, the interaction analysis had to be performed using other myosin head crystal structures. ${ }^{48}$ The crystallization of tarantula IHM has been hampered by difficulties with purifying a single homogeneous 
unphosphorylated tarantula IHM, as Ser35 monophosphorylation is constitutively present in relaxed tarantula muscle. ${ }^{24,25}$ The recent purification, crystallization, and preliminary X-ray crystallographic analysis of squid heavy meromyosin ${ }^{49}$ suggest that mollusks' IHM crystallographic structure will be available in the near future. In the second case, the closest available information for the analysis of intermolecular interactions only comes from 2.5- and 2.0-nm-resolution 3D maps with the densities of intact rapidly frozen relaxed tarantula thick filaments. ${ }^{5,23}$ A higher-resolution 3D reconstruction should be available in the future. ${ }^{45,50}$ In the meantime, we built the new tarantula model (Fig. 1), which should allow us to perform a first analysis of intra- and intermolecular interactions and their conservation. This attempt is limited by the low resolution of the 3D map, which does not allow a proper atomic level analysis, even after flexible fitting, but only allows a rough analysis of the possible residues in the general region of each interaction, as it is not possible to trace the densities of the main or side chains. Therefore, the analysis of the interactions in the model below should be considered an informed guess of the interactions that could be formed rather than a direct observation of the actual atomic contacts in a solved structure.

\section{Intramolecular interactions:}

We analyzed the intra- (Fig. 6, Suppl. Figs. 1-7) and intermolecular (Fig. 7, Suppl. Figs. 8-12) interactions using PDB 3JBH (Figs. 1-3) and by observing the peptides and charged residues involved in the interactions:

1. - Interactions “ $a$ " and " $f$ ": Interaction "a": This interaction involves residues located on S2 Ring 2, which interact with free head loop 2 (Fig. 6a, Suppl. Fig. 1, Suppl. Tables 1 and 2). Interaction "f": This interaction involves two sub-interactions: (i) Interaction "f.1" (Fig. 6a, Suppl. Fig. 2, Suppl. Tables 1 and 2) involves S2 Ring 1 and blocked head loop 2. In interaction "a," free head loop 2 interacts with Ring 2, which is negatively charged. Blocked head loop 2 was remodeled de novo and adopted a different conformation that increases the possibility that more residues will interact. The positioning of blocked head loop 2 just in front of S2 Ring 1 suggests an electrostatic interaction. $^{37}$ (ii) Interaction "f.2" (Suppl. Fig 6a, Suppl. Fig. 3, Suppl. Tables 1 and 2) involves S2 Ring 1, with blocked head H loop and $\alpha$-helix near blocked head loop 2 (Y653-H665).

2. - Interactions " $e$ " and " $g$ ": Interaction " $\mathrm{e}$ ": This interaction involves blocked head near loop 2 (K608-H622) and two blocked head regions near (before and after) the C loop with two free head ELC loops (Fig. 6b, Suppl. Fig. 4, Suppl. Tables 1 and 2). We restricted our analysis to ELC tarantula-like sequences with short NTEs (invertebrates). ${ }^{51,52}$ In our model, we cannot assess which residues are involved in making these contacts. Nevertheless, the interacting loops that are involved agree with the previously proposed loops for vertebrate smooth muscle ${ }^{35}$ (Suppl. Table 2). Interaction "g": This interaction involves the S2 at the end of the N-terminal "asymmetry zone" marked by the so-called "kink" "37 at residue N878 (M877 in cardiac chicken muscle) with the blocked head catalytic domain and the blocked head ELC (Fig. 6b, Suppl. Fig. 5, Suppl. Tables 1 and 2) through three sub-interactions: (i) free head S2 with blocked head loop NATP-V, (ii) free head S2 with blocked head ELC, and (iii) blocked head S2 with blocked head ELC C-terminal extension.

3. - Interaction "d": Interaction "d" involves two dual sub-interactions: (i) Interaction "d.1" involves the blocked head CM loop with loop NATP-II (defined here as the loop near the nucleotide ATP binding residue G173 shown in Fig. 6c, Suppl. Fig. 6, and Suppl. Tables 1 and 2). It must be noted that residues K400 (CM loop) and E165 (catalytic domain) were also proposed to interact in a previous loop remodel ${ }^{17}$ (Suppl. Table 2). The blocked head CM loop also interacts with the free head loop NATP-V and the free head near loop 2 (Y653-H665). (ii) Interaction "d.2" involves blocked head near interacting loop (I loop) ${ }^{23}$ with the free head converter domain as well as blocked head ELC C loop with the converter and relay domains (Fig. 6c, Suppl. Fig. 7, Suppl. Tables 1 and 2). 
4. - Interaction at the RLC-RLC interface: The RLCs of the blocked and free heads interact with themselves across blocked head RLC domain 1, such that the positively charged L helices are packed due to complementary charges against the blocked head domain $1,{ }^{23}$ as analyzed by Brito $e t$ $a .^{24}$

\section{Intermolecular interactions:}

Intermolecular interactions maintain IHMs suspended above the backbone surface, contacting it only in one site:

Tarantula thick filament in a relaxed state shows that the IHMs are located above the backbone surface (Fig. 1), with their S2s slightly angled $\left(\sim 6^{\circ}\right)$ and separated $\sim 2 \mathrm{~nm}$ from the surface in the "swivel" region (Fig. 3b). The model shows that the blocked head is the only part of the IHM that contacts the backbone through three backbone-anchoring intermolecular interactions - "h," "i," and " $\mathrm{j}$ "-with the two neighboring extended S2s (Fig. 3b). In other words, the IHMs are above the backbone and covalently anchored to it only through their own S2s, while the other eight interactions ("a," "h," "i," "j," and two pairs of "b" and "c") are electrostatic (Fig. 3). These suspended IHMs are interconnected, forming four coaxial helical tracks that are separated from the backbone surface and surrounded only with sarcoplasm solution molecules, which shield the IHMs electrostatically.

There are five intermolecular interactions (Figs. 2-3). Two are between adjacent IHMs ("b" and "c"; Figs. 2b and 7a), establishing the helical tracks of IHMs, and three are anchoring interactions of each IHM onto two neighbor S2s ("h," "i," and "j"; Figs. 3, 7b, c). Loops involved in the "c"5 and "b" the "h," "i," and "j" backbone-anchoring interactions are shown in Suppl. Figs. 10-12 (see also Suppl. Tables 1 and 2). In the tarantula model, the IHM-interconnecting interactions could only be used to analyze the intermolecular interactions of species exhibiting four helical tracks, like arthropods $^{5-7,53}$ and Platyhelminthes, ${ }^{12}$ as these interactions seem to be different in filaments with three ${ }^{2,3}$ or seven $^{8}$ helical tracks.

1. - Interaction " $b$ ": The free head I loop and C loop are close to the blocked head RLC NTE (Fig. 7a, Suppl. Fig. 8, Suppl. Table 2).

2. - Interaction "c": The free head CM loop is near blocked head NTE-ELC and free head loop 3 is near blocked head ELC residues K60-F67 (Fig. 7a, Suppl. Fig. 9, Suppl. Table 2).

3. - Interaction " $\boldsymbol{h}$ ": The blocked head SH3 domain is involved in an intermolecular interaction with an adjacent myosin tail (Fig. 3, Fig. 7b, Suppl. Fig. 10). The interaction "h" "holds" the blocked head SH3 domain, anchoring it on top of a neighbor myosin tail every $14.5 \mathrm{~nm}$ along each of the four helical tracks of IHMs., 23

4. - Interaction "i": This interaction occurs between the blocked head ELC and S2 of a neighbor IHM (Figs. 3b and 7b, c, Suppl. Fig. 11). Together with interaction "h," intermolecular interaction "i" can help "park" the blocked head IHM by anchoring it back onto the filament backbone.

5. - Interaction " $j$ ": This interaction occurs between the converter and relay regions of the blocked head with the S2 of a neighboring IHM. Both domains can also interact with the neighboring subfilament (Figs. 3b and 7b, c, Suppl. Fig. 12). Together with interactions "h" and "i," intermolecular interaction " $\mathrm{j}$ " can also help "park" the blocked head IHM by anchoring it back onto the filament backbone.

Analysis of conservation of interactions: 
We evaluated the conservation of specific residues involved in intra- and intermolecular interactions by performing alignments of MHC sequences (Suppl. Figs. 1-12) for 68 species (Suppl. Tables 3-5) and ordering by their MHC, ELC and RLC trees (Suppl. Figs. 13-15). According to the MHC sequence alignment tree (Suppl. Fig. 13), the MHCs of these species can be classified in two general groups: (1) striated-like and (2) smooth- and non-muscle-like. ${ }^{12}$ To analyze the conservation of intramolecular interactions (Table 1), we included species in which IHMs were detected or are currently studied by electron microscopy. The RLC-RLC interactions ${ }^{23}$ and interactions "g," "f," "e," and "d" are very important for the formation and stability of IHM. Interaction "f.2" is a key part of the formation of IHM (Figs. 2, 6), as it docks the blocked head on top of the S2, forming the precursor S2-BH (Fig. 8B). Interactions "d.1" and "d.2" are essential for binding the free head on this precursor S2-BH (Fig. 8B), which is important for IHM stability. The relative strength of these five interactions should control the stability of the assembly of the two myosin heads once the compact IHM is formed. For the analysis, we did not include interactions "a" and "f.1" (Suppl. Figs. 1, 2) because loop 2 exhibits variable length across species, and interactions "e" and "g" (Suppl. Figs. 4, 5) were too complex to be analyzed using our approach. To analyze the conservation of intramolecular interactions "d.1," "d.2," and "f.2," we defined a conservation score $\left(\mathrm{C}_{\mathrm{s}}\right.$; see Materials and Methods) that includes the conservation of the residues involved in each interaction, allowing \pm 3 residues to take into account the limited resolution of the 3D map. Scores $\mathrm{C}_{\mathrm{s}-\mathrm{d}}$ and $\mathrm{C}_{\mathrm{s}-\mathrm{f}}$ were defined to assess the conservation of interactions " $\mathrm{d}$.1," "d.2," and "f.2" in terms of IHM formation and stability. In Table 1, for each of the selected species (Suppl. Tables 3-5) we analyzed the relationship between the conservation of residues involved in critical IHM "d.1," "d.2," and "f.1"intramolecular interactions and their $\mathrm{C}_{\mathrm{s}}, \mathrm{C}_{\mathrm{s}-\mathrm{d}}$ and $\mathrm{C}_{\mathrm{s}-\mathrm{f}}$ scores in comparison with their muscle type (striated, cardiac, smooth), MHC type (striated-, smooth- or non-muscle-like), thick filament type, and IHM. In the alignments, we included isoforms a-c of myosin V, in which regulation does not involve head-head interaction, ${ }^{54}$ so the conserved residues involved in potential ionic interactions specific to myosin II could be identified, as was done previously ${ }^{17}$ (Suppl. Figs. $1-12)$.

\section{Conservation of intramolecular interactions:}

The most conserved interactions seem to be free head S2 Ring 1- loop H ("f.2") and the CM loop with the catalytic domain near loop 2 ("d.1", Y653-H665). The converter and C loop, which are also involved in the contraction process, may be less conserved depending on the muscle type, as can be seen for interaction "d.2." The sequences of the conserved myosin ATP binding site, relay, and converter are fundamentally involved in the myosin ATPase function and the production of power stroke force. The sequences of loops 2, 3, C (also called loop 4), CM, and H (Supplementary Tables 1 and 2) are involved in the myosin motor function as well as in the formation and stability of IHM and the conserved actin binding interface. ${ }^{55,56}$ Table 1 shows that the residues involved in interactions "d.1," "d.2," and "f.2" (Suppl. Figs. 3, 6-7) $\left(c f .{ }^{17}\right)$ are conserved in all MHC striatedlike sequences in invertebrates (Cs $\sim 72-85 \%$, including sponge striated MHC) and vertebrates (Cs $\sim 82-85 \%$ ), smooth-like sequences in vertebrates (Cs $\sim 88 \%$ ), non-muscle-like sequences in vertebrates (Cs $\sim 88 \%$ ), and sponge non-muscle $\mathrm{MHC}(\mathrm{Cs} \sim 63 \%)$. The Cs values for primitive nonanimal Amoebozoans (Cs 29-32\%) are close to myosin V (Cs 30-44), suggesting that their interactions are less conserved than in animals. The analysis of separate $\mathrm{C}_{\mathrm{s}-\mathrm{f}}$ and $\mathrm{C}_{\mathrm{s}-\mathrm{d}}$ contributions suggest that the residues involved in interaction "f.2," which are associated with the formation of IHM, are more conserved $\left(\mathrm{C}_{\mathrm{s}-\mathrm{f}} \sim 81-94 \%\right)$ than in interactions "d.1" and "d.2," which are associated with the stability of IHM $\left(\mathrm{C}_{\mathrm{s}-\mathrm{d}} \sim 53-85 \%\right)$.

\section{Conservation of intermolecular interactions:}

Conservation of the IHM-interconnecting interactions " $b$ " and " $c$ ": We restricted the alignment and conservation analysis of residues involved in IHM-interconnecting interactions " $\mathrm{b}$ " and "c" to 
species with long RLC NTE (Suppl. Table 5) and short ELC NTE (Suppl. Table 4) sequences. This was done because in the tarantula CPA mechanism, the activation actuator control is located in a long RLC NTE where phosphorylatable serines are located. ${ }^{26,27}$ Light chain NTEs are short or long depending on the species: RLC NTEs are long in 25 invertebrate species (and may exist at the protein or transcript level in the National Center for Biotechnology [NCBI] database) but short for the rest, while the converse is true for ELC NTEs. ${ }^{23,51,52}$ In particular, the long RLC NTE present in arthropods and Platyhelminthes has been found to be functionally involved in the destabilization of helical tracks of IHMs as a result of the CPA mechanism in tarantula thick filament. ${ }^{23-25} \mathrm{We}$ did not attempt to analyze these intermolecular interactions in vertebrate or mollusk IHMs as their 3D map interaction " $b$ " region is different from the arthropod we are studying. The actual mass density of interaction " $b$ " is clearly seen in the 3D maps of thick filaments from arthropods ${ }^{5-7,23}$ and Platyhelminthes, ${ }^{12}$ suggesting that it plays a key role in establishing the very stable helical tracks of IHMs in thick filaments. In contrast, in vertebrate and human cardiac muscle, interactions " $b$ " and "c" are only established in one of every three crowns, with only interaction " $b$ " present in the other two crowns. Interactions " $b$ " and " $c$ " both have a neutral net charge and complementary charges of the free head motor domain and adjacent free head RLC NTE in interaction "b" and the free head and adjacent blocked head ELC NTE in interaction "c" (Suppl. Table 4, Suppl. Fig. 8). Therefore, we conclude that intermolecular interactions " $b$ " and "c" are critically involved in maintaining the helical tracks of IHMs in species with long RLC NTEs and short ELC NTEs, like arthropods ${ }^{23-25}$ and Platyhelminthes. ${ }^{9}{ }^{23}$ In both interactions, the MHC side conserves specific negative and positive residues on I and C loops (interaction " $b$ ") or CM loop and loop 3 (interaction "c") as well as on the long RLC NTE (interaction "b") or short ELC NTE (interaction "c") sides (Suppl. Figs. 8-9).

Conservation of the backbone-anchoring interactions " $h$," " $i$," and " $j$ ": A quasi-atomic model of the tarantula myosin subfilaments that comprise the outer myosin layer of the thick filament backbone is not available yet, so an analysis of the backbone-anchoring intermolecular interactions "h," "i," and "j" (Fig. 7b, c) cannot be properly performed at this time. We analyzed only the tail side of these three interactions, which seem to involve several conserved negative and positive residues (Suppl. Figs. 10-12). Since in interaction "h" the SH3 domain of each IHM is located every $14.5 \mathrm{~nm}$ along each of the helical tracks, it seems plausible that alternating positive and negative charges - which are not present in myosin V, in which regulation does not involve headhead interactions - on the subfilament surface should help guide the six SH3 conserved charged residues $(\mathrm{K} 29 / \mathrm{K} 30, \mathrm{E} 37 / \mathrm{K} 38, \mathrm{~K} 67$, D69) for docking the blocked head back on the backbone of the thick filament (Suppl. Fig. 10). The presence of significantly longer NTEs in all the SH3 of myosin II-which is not present in myosin V-suggesting that it is involved in blocked head docking and establishing the helical tracks of IHMs. Our analysis of interaction " $i$," which is restricted only to species with short ELC NTEs (Suppl. Fig. 11), shows that six negative (E79, E84, D85, E88, D94, E140) and four positive (K78, K91, K95, K144) residues are highly conserved and may make contact with subfilaments. The analysis of interaction "j" (Suppl. Fig. 12) shows that in contrast to myosin Vs, most myosin IIs have two negative (E744, D504) and four positive (K721, R758, H488, K499) residues that are very conserved in the converter and relay. We conclude that some specific residues involved in the intermolecular interactions " $h$," " $i$," and " $\mathrm{j}$ " are crucially involved in the axial and lateral docking of the IHMs on the thick filament backbone, and are conserved in most myosin II sequences for interactions " $h$ " and " $\mathrm{j}$ " and in short ELC NTE sequences for interaction "i."

In summary, the oppositely charged and conserved residues involved in the five intramolecular interactions maintain the IHM in a compact off structure, explaining the conservation of the motif across animal species. The oppositely charged residues involved in the five intermolecular interactions retain the IHMs, making helical tracks of myosin heads on the surface of the backbone, and are conserved in arthropods and Platyhelminthes. 


\section{Discussion}

The structure of tarantula thick filaments has been extensively studied. ${ }^{5,53,57,58}$ These filaments exhibit four helical tracks of myosin heads ${ }^{53}$ (compacted as IHMs) ${ }^{5,23}$ above the backbone surface. Since the tarantula IHM crystal structure had not been determined, the quasi-atomic model PDB $3 \mathrm{JBH}$ (see Results) allows for provisional analysis of its intra- and intermolecular interactions, as well as building a quasi-atomic model of the complete tarantula myosin molecule (including its tail), a subfilament model, and the backbone formed by a ring of twelve subfilaments around the paramyosin core (Alamo et al. in progress). The tarantula myosin RLC NTE, with two phosphorylation sites at Ser35 and Ser45, has been shown to be crucial for the relaxation and activation of tarantula thick filaments, ${ }^{23}$ for which we have proposed a tarantula CPA mechanism ${ }^{24}$ ${ }^{27}$ (Fig. 8C-F). This dual phosphorylation mechanism could be extended to other chelicerate striated muscles, like that of Limulus ${ }^{6}$ and scorpion, ${ }^{7}$ and possibly other arthropods with thick filaments that exhibit 4-stranded helical tracks of IHMs together with myosin RLCs with a long NTE and two phosphorylatable serines. In contrast, a different activation mechanism is present in vertebrate skeletal $^{28,29}$ and cardiac muscle, ${ }^{30,31}$ which have thick filaments that exhibit a perturbed 3-fold helical array of IHMs, as well as in vertebrate smooth muscle, ${ }^{32}$ which also has IHMs but with a proposed ELC activation role, ${ }^{33}$ and mollusks, which have 7-fold helical tracks of IHMs with ELC direct $\mathrm{Ca}^{2+}$-binding activation control. Below we discuss how the intra- and intermolecular interactions associated with the tarantula model (Figs. 2b, 6, 7) are sequentially established, forming the IHMs, and how the helical tracks of IHMs in a relaxed state are sequentially disrupted upon activation and sequentially reformed upon relaxation (Fig. 8, "interaction table"). This discussion is based on the tarantula CPA mechanism and aims to explain the structural basis of the ATP turnover rates detected in tarantula SRX. ${ }^{22}$

\section{Interactions form IHMs and their helical tracks in a relaxed state:}

How are tarantula thick filaments formed in a relaxed state?: The intramolecular interactions are present in isolated switched-off myosin II molecules, which form IHMs in a solution. ${ }^{17}$ Myosin subfilaments are formed by the coiling-coil tails of three myosin molecules with one pair of heads protruding every $43.5 \mathrm{~nm}$. Twelve myosin subfilaments are packed around a paramyosin core, forming the filament backbone (Fig. 1). Initially, the myosin S2s emerge helically over the backbone (Fig. 2b) and the pairs of heads are disordered and protruding, not yet forming IHMs (precursor IHMs; Fig. 8A). These heads are in the relaxed closed conformation (Fig. 4) with RLC NTEs that are not phosphorylated. The interactions table in Fig. 8A-C shows the four stages in which the intra- and intermolecular interactions could be sequentially involved, establishing the fully functional IHMs (Fig. 8C) and forming the helical relaxed structure of the thick filament: (1) Only one head in each pair can establish the "h," "j," and "i" intermolecular interactions (Figs. 3, 7) with the neighbor myosin tail, docking itself as a blocked head closed conformation (Fig. 4) by establishing the intramolecular interactions "g" and "f" with the S2 (Fig. 8B). (2) Once this blocked head is docked, the partner free head, which is also in a closed conformation (Fig. 4), can dock (Fig. 8B). (3) A temporarily activated protein kinase $\mathrm{C}$ can then permanently (i.e. constitutively) phosphorylate ${ }^{24,}{ }^{25}$ RLC NTE at its target Ser35 (i.e. the exposed Ser35 of free heads, since the Ser35 of blocked heads are not sterically accessible ${ }^{23-25}$ (Fig. 8C). (4) Ser35 monophosphorylation of the free heads allow them to sway away and back according to Brownian motion due to the breaking and reforming of the RLC-RLC intramolecular interactions "e," "d," and "a," which are required for assembling an IHM (Figs. 4, 8C). Free head swaying is supported by several lines of evidence from our lab. ${ }^{23-27}$ Negatively stained isolated smooth muscle myosin IHMs appear to show free heads as more mobile, detaching from their partner blocked heads, and with their motor domains adopting different orientations. ${ }^{59}$ In addition, X-ray diffraction studies show that a fraction of constitutively on motors allow the muscle to immediately respond to calcium activation ${ }^{60}$ (cf.). ${ }^{24}$ 


\section{Intramolecular interactions are involved in forming IHMs:}

Intramolecular interaction "f.2," which is involved in the formation of the IHM, is more conserved in all the studied animal species than in non-animal species: The IHM is ubiquitous. Table 1 shows that the IHM is present in all animal species with muscles, irrespective of the muscle type (striated skeletal, cardiac, or smooth), filament type (bipolar, side-polar) or MHC type (striated-, smooth-, or non-muscle-like). The wide presence of IHM correlates with the conservation of residue charge in the "d.1," "d.2," and "f.2" interactions in all animal species shown in Table $1\left(\mathrm{C}_{\mathrm{s}}>\sim 63 \%\right)$. The ranges of conservation $-\mathrm{C}_{\mathrm{s}-\mathrm{f}} \sim 40-94 \%$ and $\mathrm{C}_{\mathrm{s}-\mathrm{d}} \sim 24-85 \%$ - for these critical interactions on MHC II suggest matched variations in the formation and stability of the IHM as these species evolved. On one hand, the absence of IHM in the Amoebozoan Acanthamoeba castellani correlates with a smaller conservation percentage $\left(\mathrm{C}_{\mathrm{s}} \sim 32 \%\right)$, suggesting the importance of these critical interactions in the formation and stability of the IHM. On the other hand, the chicken vertebrate smooth muscle head-tail interaction is likely the most stable interaction as it establishes the motif structure ${ }^{35}$ with the smallest rate of product release ${ }^{61}$ and results in a higher conservation percentage of the residues involved in the formation and stability of the IHM $\left(\mathrm{C}_{\mathrm{s}-\mathrm{f}}\right.$ and $\left.\mathrm{C}_{\mathrm{s}-\mathrm{d}} \sim 94 \%\right)$. Also, studies with isolated $10 \mathrm{~S}$ myosin II HMM from smooth and non-muscle-like MHC sequences suggest that the interaction between the blocked head and S2 is required for the formation of a stable IHM. ${ }^{17,}{ }^{61}$ This agrees with the similar residue conservation values in vertebrate smooth $\left(\mathrm{C}_{s-\mathrm{f}} \sim 94 \%\right)$ and non-muscle-like MHC sequences $\left(\mathrm{C}_{\mathrm{s}-\mathrm{f}} \sim 94 \%\right)$. Finally, it has been suggested that the strength of the intermolecular interactions is weaker in unregulated myosin, showing that IHMs are less common in solution than regulated myosin. ${ }^{17}$ This is in accordance with the smaller conservation values $\left(\mathrm{C}_{\mathrm{s}-\mathrm{d}} \sim 78-82 \%\right)$ in vertebrate cardiac muscles from mice, humans, and zebra fish. The consistency of the results among species that was achieved with this interaction scoring approach reassured our confidence in our interaction analysis, in spite of the clear resolution limitations of the 3D map and the lack of definition of the precise residue contacts that are involved in the intramolecular interactions. We conclude that "d.1," "d.2," and "f.2" intramolecular interactions are critically involved in the formation and stability of the IHM. The conservation of residues involved in these interactions support the conservation of the IHM across all animal species, irrespective of the presence of muscles (like in sponges which lack muscle), muscle type, filament type, or MHC II type. The conservation analysis of these interactions suggests they are more conserved in animals than in primitive non-animals, supporting our conclusion-which is based on the fact that the IHM was detected by electron microscopy ${ }^{14}$ - that IHMs evolved after Amoebozoa and animals diverged.

\section{Intermolecular interactions are involved in the formation of the helical tracks of IHMs along the filaments:}

For arthropods like tarantulas, in which activation is dependent upon phosphorylation, the two intermolecular interactions " $b$ " and "c" and the remaining anchoring interactions "h," "i," and "j" (Figs. 3, 6, 7) maintain the stability of the helical tracks of IHMs more than in vertebrates or mollusks.

IHM interconnecting interactions: The "b" and "c" intermolecular interactions are critically involved in the maintenance of the helical tracks of IHMs in species with long RLC NTEs and short ELC NTEs, such as arthropods ${ }^{23-25}$ and Platyhelminthes. ${ }^{9,}{ }^{23}$ In both interactions, the MHC side conserves specific negative and positive residues on I and C loops (interaction "b") or CM loop and loop 3 (interaction "c") as well as on the long RLC NTE (interaction "b") or short ELC NTE (interaction "c") sides (Suppl. Figs. 8-9). This analysis could be extended to the similar thick filaments of the Platyhelminth Schistosome.

Backbone anchoring interactions: Some specific residues involved in these intermolecular interactions are crucially involved in the axial and lateral docking of IHMs on the filament 
backbone, which are conserved in most myosin II sequences (interactions " $h$ " and "j") and short ELC NTE sequences ("ii").

MHC type controls filament type formation: According to the average distance tree for MHC II sequence alignment (Suppl. Fig. 13), MHCs can be classified into three types: striated-, smooth-, and non-muscle-like. Table 1 shows that the MHC type controls filament type formation: striatedlike MHC form helical bipolar thick filaments with various numbers of helical tracks (3-7), while smooth-like MHC form side-polar thick filaments and non-muscle-like MHC form bipolar minifilaments.

Implications of myosin interactions in the evolutionary origin of the switched off state: This state may have arisen early in species' evolution as a means of switching off non-muscle myosin. Nonmuscle myosin II is monomeric in an off state, so only intramolecular interactions between heads would have been possible. As muscles evolved, they may have retained these interactions. In filaments, additional (intermolecular) interactions became possible and advantageous in an evolutionary sense. Thick filaments could be formed first on paramyosin cores, allowing various numbers of helical tracks of IHMs: seven in mollusk striated muscle; four in arthropod and Platyhelminth striated muscle; and three in vertebrate skeletal or cardiac muscle when the paramyosin core was lost. Additionally, IHM regulation could also have evolved from being controlled by direct ELC $\mathrm{Ca}^{2+}$ binding (mollusks), RLC phosphorylation (in arthropods and possibly in Platyhelminthes) or being unregulated in vertebrates.

\section{On activation interactions are disrupted, disassembling IHMs and their helical tracks:}

The "interactions table" in Fig. 8C-F shows which intra- and intermolecular interactions are established in a relaxed state (Fig. 8C) and which interactions are progressively removed after a relaxed tarantula thick filament is activated (Fig. 8D-F), such that helical tracks of IHMs are disassembled, with the disordered free and blocked heads protruding away from the backbone (Fig. 8D-F), as observed by negative staining ${ }^{62}$ and equatorial X-ray diffraction. ${ }^{63}$ We have shown that the structural differences and location within the IHM could preset the order in which the free and blocked head are released upon phosphorylation, having the free head the right conformation (Fig. 4) to be released first to interact with actin, followed by the blocked head (if required) ${ }^{24,25}$.

\section{Interactions sequentially reform, comprising IHMs and their helical tracks upon relaxation:}

We propose that the disordering of myosin heads produced by activation (Fig. 8C-F) is restored after relaxation in three independent steps, depending on which step the motif (Fig. 8F-C) is:

Formation of precursor blocked head-S2 motif: Once the monophosphorylated Ser45 of a blocked head is dephosphorylated (Fig. 8E-D, top IHM), the disordered blocked head can eventually reestablish intramolecular interactions "g" and "f" with its own S2 (Fig. 8D, top IHM), reattach to the two neighbor S2s (Fig. 7) by re-establishing the anchoring intermolecular interactions "h," "j," and "i." This allows the blocked head to be docked back and anchored precisely in the correct axial position (i.e., every $14.5 \mathrm{~nm}$ ). It has been reported that interaction between the blocked head and its own S2 is needed to form a stable IHM. ${ }^{17,61}$ These intramolecular interactions can be established between a blocked head and its S2 in isolated HMM molecules, in contrast to the intramolecular interactions " $h$," "i," and "j," which can only be established in a filament.

Reassembly of IHM from a blocked head-S2 precursor motif: Once the partner diphosphorylated free head of a docked blocked head is Ser45 dephosphorylated by myosin light chain phosphatase, again becoming a Ser35 monophosphorylated swaying free head (Fig. 8D-C, middle IHM), they could eventually dock back onto the blocked head-S2 precursor motif by re-establishing first the closer RLC-RLC interactions between both blocked and free heads and then the remaining 
intramolecular interactions "e," "d," and "a." This reassembly should be guided by the complementary charges of domain 1 of the blocked and free head RLC NTEs, as we have proposed. $^{24}$ Thus, the RLC domain 1 seems to be better conserved than the rest of the RLC domains. $^{64}$

Formation of helical tracks of IHMs: When a similar process occurs in axially adjacent reassembled IHMs, pairs of "c" and "b" interactions can be re-established between them (Fig. 1), stabilizing the helix first in short patches and then so on. This finely tunes the formation of helical tracks of IHMs with a subunit distance of $14.5 \mathrm{~nm}$, been the $43.5 \mathrm{~nm}$ helical repeat enforced by the twelve myosin subfilament symmetry.

\section{Implications of IHM interactions on smooth muscle:}

It was striking to find that vertebrate smooth muscle $\mathrm{IHM}^{11,34,35}$ was present in invertebrate striated muscle ${ }^{5}$ with similar IHM models (PDB $1 \mathrm{I}_{84^{35}}$ vs. PDB 3DTP ${ }^{23}$ ). Also, the thick filaments present in invertebrate striated muscle ${ }^{5}$ were similar to invertebrate smooth muscle ${ }^{12}$ with similar IHMs. In addition, a striking similarity between the myosin II RLCs of Schistosome and tarantula was observed: both RLCs exhibit a long NTE with two putative phosphorylatable serines, ${ }^{23,}{ }^{24}$ suggesting that our present analysis in this paper could be applied to the smooth muscle IHM of Platyhelminthes. Concerning vertebrate smooth muscle, which has an unknown specialized sidepolar thick filament structure, we cannot infer how the specific IHM interactions in vertebrate smooth muscle could be involved in assembling non-helical side-polar filaments Trybus et al. ${ }^{61}$ have discussed in detail the need for vertebrate smooth muscle to have two myosin heads, headhead interactions, and S2 for regulation by RLC phosphorylation. We can only mention that S2, which is suggested to mediate specific interactions with the head that are required to achieve an off state in vertebrate smooth and non-muscle myosin $\mathrm{II}^{61}$ and is essential for regulation, ${ }^{61}$ should be involved in IHM intramolecular interactions similar to "a," "f," and "g." Supporting Trybus et al., ${ }^{61}$ our results with tarantulas suggest that these interactions could correspond to interactions "a," "f," and perhaps "g," explaining the necessity of a minimal length of S2 for regulation. ${ }^{61}$ Two of these interactions, "a" and "f," are involved in the interaction of S2 Rings 2 and 1 with free head loop 2 and blocked head, which is part of myosin II regulation in smooth and skeletal muscle. ${ }^{42}$

\section{Structural basis of the tarantula muscle SRX state:}

The two heads in the IHM have inhibited ATPase: the blocked head exhibits a closed conformation $^{23}$ of the nucleotide pocket switch 2 (Fig. 4), preventing phosphate release, ${ }^{46,47}$ and it is "parked," or locked-in, to the backbone (Fig. 8C) with ATP activity that is sterically "blocked," as its actin-binding interface is positioned on the converter domain of its partner free head. This inhibits ATPase activity by stabilizing the converter domain movements needed to release phosphate. ${ }^{11}$ Relaxed vertebrate skeletal and cardiac muscle fibers exhibit a slow ATP turnover rate. ${ }^{18,19}$ Naber et $a .^{22}$ proposed that this is an adaptation to save energy in animals like tarantulas that spend long periods immobile while poised to quickly capture prey. This state-which strongly inhibits myosin ATPase activity and has been observed in a variety of muscle types - was called the SRX state in the pioneering work of Cooke et al. ${ }^{20}$ Its importance in muscle has been described by many authors. ${ }^{18-20,22,65}{ }^{66}$ Structurally, SRX has been ascribed to the IHM. ${ }^{18,20}$ In relaxed tarantula muscle, three rates are detected: ${ }^{22}$ very slow ( $\left.>1800 \mathrm{~s}\right)$, slow $(250-300 \mathrm{~s})$, and fast $(<30 \mathrm{~s})$. Based on the PDB 3JBH model, its interactions, and the tarantula CPA mechanism ${ }^{14,24,26,27}$ (Fig. 8C-F), we ascribe the very slow rate to the docked unphosphorylated heads, the slow rate to phosphorylated docked heads, and the fast rate to phosphorylated undocked heads. Therefore, as shown in Fig. 8C and explained below, the docked (helically ordered) unphosphorylated blocked and a few free heads, which both have a very slow rate, and the transiently docked Ser35 monophosphorylated free heads, which have a slow rate, are responsible for the two SRX levels in tarantulas, ${ }^{22}$ while the 
remaining (disordered) swaying free heads with Brownian motion exhibit the fast rate detected in the disordered relaxed state.

Structural basis of the very slow, slow, fast, and very fast rates in tarantula striated muscle:

(1) Very slow rate origin: The very slow rate is ascribed to unphosphorylated blocked heads and the few unphosphorylated Ser-35 free heads docked in the IHM motif. The ATPase rate is determined by the strength of the intramolecular interactions, and since this muscle is thick-filament-regulated, some other mechanism momentarily undocks or activates the myosin. The very slow rate is expected to exist in other thick-filament-regulated filaments. In relaxation, all blocked heads are unphosphorylated, as endogenous myosin light chain phosphatase is active, dephosphorylating any Ser45 mono-phosphorylated blocked heads. The blocked head rate is very slow, as its ATPase activity is sterically "blocked"11 and locked in to the backbone. ${ }^{26,27}$ This "parking," or locking in, of half the available myosin heads in a close conformation is an effective mechanism for saving energy by preserving bound ATP.

(2) Slow rate origin: The slow rate is ascribed to docked swaying Ser35 monophosphorylated free heads (Fig. 8C), for which ATP activity is transiently inhibited while docked in the IHM motif.

(3) Fast rate origin: The fast rate is ascribed to undocked swaying Ser35 monophosphorylated free heads (Fig. 8C) and interpreted as a way to enable at least half of the available heads to explore as structural sentinels if there are any activated thin filaments, to save energy, and to make a faster transition to active states if there are activated thin filaments nearby, favoring quick force production like in single twitches or twitch summation. ${ }^{24}$ The Ser35 monophosphorylation is constitutively (i.e., permanently) present on most free heads, suggesting that it is a simple way to segregate heads that are better located to quickly sway away by Brownian motion and interact with $\mathrm{Ca}^{2+}$-activated thin filaments. ${ }^{24}$

(4) Very fast rate origin: In contrast to the relaxed state (Fig. 8C), remnants of these three heads' populations (Fig. 8D-E) briefly coexist in an activated state (Fig. 8D-F) together with a quickly increasing fourth population that is formed by released swaying Ser35-monophosphorylated free heads, Ser45-monophosphorylated blocked heads, and diphosphorylated free heads, which are in the transition pre-power stroke conformation ${ }^{23}$ (Fig. 4) and are activated when bound to an activated thin filament located $\sim 5 \mathrm{~nm}$ away. ${ }^{46}$ This population is associated with the very fast time constant $(<$ $0.1 \mathrm{~s}$ ) detected in active muscle fibers ${ }^{18}$ (Fig. $8 \mathrm{~F}$ ), as a high rate is required after activation during muscle contraction.

In relaxed tarantula thick filaments, a "closed" conformation that prevents phosphate release is required for the formation of helical tracks of IHMs on the backbone. ${ }^{46}$ Blebbistatin, which inhibits myosin II ATPase activity, ${ }^{67}$ stabilizes helical tracks in a relaxed state by promoting a closed state. ${ }^{68}$ These two results suggest that in tarantula blebbistatin should closely hold the two heads on IHMs, diminishing the free heads that sway away and implying a very slow rate. In fact, blebbistatin stabilizes the SRX state in skeletal muscle, producing a very long-lived myosin-nucleotide complex with a very slow time constant, ${ }^{65}$ which favors our interpretation of the very slow rate in tarantulas. Additionally, in the presence of blebbistatin, the spin-labeled nucleotides bound to myosin have an oriented spectrum in SRX in both slow and fast skeletal muscle, similar to those observed in relaxed tarantula fibers without blebbistatin. ${ }^{65}$ This strongly supports the proposed structural origin of the SRX state (Fig. 8).

Comparison of the SRX states of vertebrate skeletal and cardiac muscle:

In contrast to tarantula muscle, in which very slow (>1800 s), slow (250-300 s), and fast (<30 s) rates are detected, ${ }^{22}$ in vertebrate skeletal ${ }^{18}$ and $\operatorname{cardiac}^{19}$ muscle only slow (230 and 138-144 s) and 
fast rates are detected. Relaxed vertebrate and tarantula muscle have several structural and functional differences. Thick filaments from tarantulas exhibit four helical tracks of IHMs with similar motifs on the three crowns of a $43.5 \mathrm{~nm}$ repeat, while vertebrate and cardiac muscle exhibit three perturbed helical tracks in which only two crowns exhibit similar motifs. Additionally, cardiac muscle features the cMyBP-C zone. Tarantula muscle has thick filament activation, while in vertebrate skeletal and cardiac muscle, the thick filament acts as a modulator. This "locks in" the tarantula unphosphorylated blocked head to the very slow rate and causes it to become swaying blocked head (inducing the slow rate) only after becoming Ser45 monophosphorylated blocked head by a myosin light chain kinase, ${ }^{23-27}$ which can only occur upon $\mathrm{Ca}^{2+}$ activation. In vertebrate skeletal and cardiac muscle, in which thick filaments are not regulated, the blocked heads sway without phosphorylation, eliminating the very slow rate. Studies using bifunctional fluorescence labels that are bound to intact vertebrate skeletal muscle myosin unphosphorylated RLC to quantify the orientation of the myosin lever arm by obtaining polarization measurements revealed three preferred orientations in the relaxed state: two with a long axis that is roughly parallel to the filament axis, and one that is roughly perpendicular. ${ }^{28}$ In vertebrate skeletal muscle RLC, phosphorylation disrupts the parallel orientation, shifting the equilibrium toward a perpendicular orientation, which permanently releases phosphorylated free and blocked heads. Similar studies on vertebrate cardiac muscle, in which almost no endogenous (constitutive) Ser15 monophosphorylation was detected, revealed that in a relaxed state (with unphosphorylated RLCs), there was a conformational equilibrium between parallel and perpendicular states and Ser15 monophosphorylation destabilized the parallel conformations, promoting a perpendicular conformation. ${ }^{31}$ We believe that the two preferred parallel orientations are associated with the slow rate of docked blocked and free head (SRX) in the IHM and that the perpendicular orientation is associated with the fast rate in undocked swaying free and blocked heads, as the free and blocked heads are active and can sway away without being monophosphorylated. Therefore, in contrast to tarantula relaxed muscle, SRX in vertebrate relaxed muscle should ascribe the only detected rate (slow) to both swaying blocked and free heads without permanently docked blocked heads.

\section{Conservation of the SRX state:}

The SRX state has been detected in arthropod striated muscle ${ }^{22}$ and vertebrate skeletal (rabbit psoas and soleus ${ }^{18}$ ), cardiac (rabbit, ${ }^{19}$ human [Drs. James W. McNamara and Roger Cooke, personal communication]) and smooth muscle (preliminary result; Drs. Ed Pate and Roger Cooke, personal communication). The conservation of critical intramolecular interactions across animals and IHM structure across bilaterians (Table 1) suggests that SRX should be a conserved fundamental feature of IHM to save ATP.

In conclusion, the tarantula quasi-atomic model PDB 3JBH, its intra- and intermolecular interactions, and the cooperative-phosphorylation activation (CPA) mechanism show the structural basis of SRX in tarantula muscle and its differences from vertebrate muscle. IHM conservation reveals that intramolecular interactions, which maintain the asymmetric structure of IHM, are crucial for maintaining muscle relaxation in animal species.

\section{Materials and Methods}

\section{Characterization of full-length cDNA of tarantula MHC:}

Aphonopelma MHC sequencing follows the approach of Zhu et al. ${ }^{38}$ The total length of the sequenced cDNA is $6611 \mathrm{bp}$, with a $5862 \mathrm{bp}$ open reading frame that begins at position 126. The deduced residue sequence GenBank KT619079, which is comprised of 1953 residues, contains characteristic features of ATP-binding and actin-binding sites of myosin. A simple comparison with fully sequenced heavy chains shows that $50-64 \%, 31-35 \%$, and $30 \%$ identity with sarcomeric (vertebrate striated and cardiac muscles and invertebrate muscles), non-sarcomeric (smooth 
muscle), and unicellular (Dictyostelium discoideus) MHCs, respectively. The myosin head region is more conserved (57-69\% and 49-50\% identity with sarcomeric and non-sarcomeric MHCs, respectively) than the coiled-coil rod (43-62\% and $26 \%)$.

\section{Image processing and $3 \mathrm{D}$ reconstruction}

The 3D map used for flexible fitting of the tarantula homology model was EMD-1950 with a 2.0 $\mathrm{nm}$ resolution, the same 3D map we used for IHM PDB 3DTP. ${ }^{23}$

\section{Homology modeling}

\section{Modeling of ELC:}

The tarantula Aphonopelma sequence was submitted to the BioInfoBank Meta Server (http://meta.bioinfo.pl/submit wizard.pl). From all the models we received, those with the higher scores were chosen. From these, the model with all the residues in the sequence was selected: PDB 2OVK. Two copies were made, one for the free head and another for the blocked head, and they were superposed on the previous IHM structure (PDB 3DTP) by the Chimera MatchMaker tool ${ }^{69}$.

RLC modeling:

The tarantula Aphonopelma sequence was submitted to the SwissModel Server to obtain the homology model of the RLC (http://swissmodel.expasy.org/) using the PDB 3DTP chains E and F as a template.

Modeling of MHC (Motor domain and S2):

The sequences that correspond to the motor domain and S2 of PDB 3DTP were extracted from the full-length tarantula Aphonopelma MHC sequence. These 965 residues were aligned to the sequence of PDB 3DTP chains A and B (free and blocked head MHC sequences) and submitted to SwissModel Server to obtain the corresponding free head and blocked head homology models. The final model was assembled and saved as a PDB file using Chimera. ${ }^{69}$

\section{Flexible fitting}

Flexible fitting was performed in seven steps: (1) Initial preparation of the model (assembly and minimization): An initial minimization of the structure was performed to avoid problems during the flexible fitting procedure, as the chains came from different sources. Using VMD, ${ }^{70}$ an explicit water box was generated with the VMD Automatic PSF Builder. Ions were placed in a separate step using $0.15 \mathrm{M}$ of salt. Then, the solvated and ionized structure was minimized by 2000 steps using NAMD. ${ }^{71}$ To estimate the effect of the minimization, the amounts of clashes before and after the minimization procedure were calculated with Chimera. Before minimization, 2757 clashes were detected, and after minimization, the number of clashes decreased to 84. (2) Initial rigid body docking: The minimized model was fitted as a rigid body to the tarantula 3D map with the Situs qrange tool. (3) Preparing the PDB file for flexible fitting: The PDB file was edited and converted to a suitable format for X-PLOR ${ }^{72}$ refinement. Internal water molecules were added to empty cavities to stabilize the structure during flexible fitting using the Dowser program. ${ }^{73}$ (4) Flexible fitting using Situs (2.4) and Sculptor (1.1.6): Codebook (CB) vectors were created for the structure using Situs. ${ }^{74}$ The generated connectivity among CB vectors was edited to remove some bonds and provide the network with flexibility. The optimal connectivity network for the map was interactively chosen using the Sculptor (1.1.6) graphic interface. Finally, the flexible fitting step was performed using X-PLOR. (5) Full water box minimization of the flexibly fitted model: To reduce clashes in the flexibly fitted model, we performed a final minimization in the same way as the initial minimization. (6) Building two adjacent HMMs: The IHM model was duplicated and the 
copy was helically placed at the next upper crown to generate a model with two interacting HMMs that allow intermolecular interactions to be studied. (7) Remodeling of the interacting loops: To seek possible residues that can create interactions, we used X-PLOR scripts to allow the lateral chains of interacting loops to move freely in order to find the residues that can be located at the atomic contact distance range.

\section{Bioinformatics analysis:}

We used JalView (ver. 2.8) ${ }^{75}$ to analyze the sequences retrieved from the UniProt database (http://www.uniprot.org/). The multiple sequence alignment was performed with Clustal (ver. 2.0 ${ }^{76}$ using default parameters and ordered according to the average percent identity in a distance tree (Suppl. Figs. 13-15). For the conservation analysis shown in Suppl. Figs. 1-12 and Table 1, we chose the MHC sequences reported for non-muscle, smooth, skeletal, striated, and cardiac muscle of the 68 species shown in Suppl. Table 3. Myosin V sequences are included for comparison, as they do not establish IHM. ${ }^{17}$ In addition, we chose to align the myosin 28 RLCs and 62 ELCs sequences, as shown in Suppl. Tables 4 and 5. In Table 1, we analyzed the conservation of the residues involved in "d.1," "d.2," and "f.2" intramolecular interactions by ascribing a score value to each sub-interaction (d.1 i, ii, iii; d.2 i, ii, iii; f.2 i, ii, iii) as a percentage. In the sequence alignment, each species was compared to the corresponding tarantula sequence (which was considered 100\%). The score was 100\%: 1) if both sides of the sub-interaction were composed by the same number of conserved residues and had opposite charges; 2) if the sides of the interactions had a different number of residues, all of which were conserved; 3 ) if the sides of the sub-interactions had a different number of residues, one residue that was not conserved, and the same number of conserved residues on each side. Otherwise, the score was $75 \%, 50 \%$, or $25 \%$ if the conservation was present in the previous/following residue in the sequence $(+/-1,2$, or 3 amino acids, respectively) or $0 \%$ if the difference in position was greater than three amino acids, there was no conservation on one side of the interaction, or a residue with the opposite charge was in the same position. We calculated the final conservation score, $\mathrm{C}_{\mathrm{s}}$, for each species as the average of the "d.1," "d.2," and "f.2" sub-interactions as well as the scores for the contributions of "d.1" and "d.2" $\left(\mathrm{C}_{\mathrm{s}-\mathrm{d}}\right)$ or "f.2" $\left(\mathrm{C}_{\mathrm{s}-\mathrm{f}}\right)$. Only complete sequences with evidence at the transcriptional or protein level were selected for the analysis. For anemone, we only used the few available MHC sequence fragments for different anemone species that are involved in the "d.1," "d.2," and "f.2" intramolecular interactions.

\section{SAXS analysis:}

The sample preparation and data collection details for squid HMM were reported in a previous publication. ${ }^{15}$ Scattering intensity, I(q), is in arbitrary units, with $q=4 \pi \sin (\theta) / \lambda$ in units of $\AA^{-1}$. The scattering angle is $2 \theta$ with a wavelength of $\lambda=1.2563 \AA$. Scattering profiles for PDB 3DTP and $3 \mathrm{JBH}$ were computed using FoXS ${ }^{77}$ and $\mathrm{CRYSOL}^{78}$ to check consistency, especially at scattering angles beyond $q=0.3 \AA^{-1}$. CRYSOL required more than the default number of harmonics (30) for agreement with FoXS. There were some systematic baseline shifts between the two algorithms at $\mathrm{q}$ $>0.3 \AA^{-1}$ (not shown), but both algorithms agreed on the location and magnitude of the difference between the PDB models. Only FoXS results are reported here. FoXS was allowed to fit both the computed profiles to the squid HMM experimental data using the customary parameters of hydration layer, excluded volume, and background adjustment. ${ }^{15}$

\section{Accession numbers:}

The tarantula (Aphonopelma sp.) myosin $\mathrm{ELC}^{38}, \mathrm{RLC}^{38}$ and $\mathrm{MHC}$ sequences are included in the supplementary data and have been deposited into the GenBank ${ }^{39}$ with accession numbers KT390185, KT390186, and KT619079. Two adjacent HMM tarantula myosin II IHMs have been 
deposited in the Research Collaboratory for Structural Bioinformatics PDB with the entry code 3JBH.

\section{Acknowledgments}

We thank Dr. Roger Craig for his critical reading, Dr. Guidenn Sulbarán for her comments, Dr. Gustavo Márquez for his help with the manuscript, and Mr. Jorge Basualdo (ULA) for his initial help in this project. The images in Figs. 1-4, 6, and 7 and Suppl. Figs. 1-12 were made using UCSF Chimera software. ${ }^{69}$ Cornell High Energy Synchrotron Source (CHESS) is supported by the NSF \& NIH/NIGMS via NSF award DMR-1332208, and the MacCHESS resources are supported by NIGMS award GM-103485 (to R.E.G.). This work was supported in part by NIH grant R01GM62968 U.S.A. (to W.W.) and the Howard Hughes Medical Institute, U.S.A. (to R.P.).

We dedicate this paper to the memory of Drs. Hugh E. Huxley and Andrew Szent-Györgyi.

\section{Supplementary material}

Supplementary material associated with this article can be found in the online version at http://

\section{Figure legends}

Fig. 1. Wide-eye stereo pair of the longitudinal view of the 3D reconstruction of the frozenhydrated tarantula thick filament, filtered to 2-nm resolution (EMD-1950) ${ }^{23}$ and showing four helical tracks of interacting-heads motifs (IHMs; blue), twelve myosin subfilaments (gray) and the paramyosin core (orange). The 3D map segment shows four 14.5-nm crowns, each of which has four IHMs. The quasi-atomic model PDB 3JBH (formed by two IHMs), which is shown as spheres in the right helix, was flexibly fitted to the 3D map (see Materials and Methods). The myosin heavy chain (MHC) of the blocked head $(\mathrm{BH})$ and free head $(\mathrm{FH})$ are shown in green and blue. The two myosin essential light chains (ELC) are in magenta $(\mathrm{FH})$ or orange $(\mathrm{BH})$. The two myosin regulatory light chains (RLC) are in red (FH) or yellow (BH). Bar: $14.5 \mathrm{~nm}$.

Fig. 2. Wide-eye stereo pairs of the 2-nm resolution 3D reconstruction of frozen-hydrated relaxed thick filament of tarantula (EMD-1950), ${ }^{23}$ shown in grey, with the flexibly fitted quasi-atomic model PDB 3JBH, as viewed from the front (a) or back (b) of the filament surface. The model includes the densities where several loops are located in the blocked head region of interactions " $\mathrm{b}$ " and "c" (see Materials and Methods). The MHC in PDB 3JBH shows six surface loops (2, H, CM, $3, \mathrm{C}$, and I) that are involved in the interactions. Also, the ELC in the PDB 3JBH model shows the extra two amino acids that are missing in the chicken ELC sequence. In (b), the intramolecular interactions are: "a" (FH MD loop 2-S2), "d" (FH MD-BH MD), "e" (FH ELC-BH MD), "f" (S2BH MD) and "g" (S2-BH ELC). The intermolecular interactions are: "b" (BH RLC-FH MD) and "c" (BH ELC-FH MD), which are established with the adjacent IHM in the filament, and "h," which occurs between the blocked head SH3 domain and a neighbor myosin S2 (shown as a 2-nm pink cylinder). Each of these interactions is shown in Figs. 6 and 7. MD: motor domain of the myosin head. See legend of Fig. 1. Bar: 50 Å.

Fig. 3. (a) Wide-eye stereo pairs of three adjacent IHMs forming part of a helix. The intermolecular interactions " $b$ " and "c" are shown with the RLC and ELC of the neighboring blocked head regulatory domain, and interaction " $h$ " is shown with the neighboring S2. The surface of the 3D map corresponding to the IHM in the center is highlighted in yellow. For clarity, the S2 of the model of the two left IHMs has been extended as coiled-coil $\alpha$-helices (pink). Since the subfilament structure is not known, the two neighboring subfilaments are depicted as cylinders with diameters of about $2.2 \mathrm{~nm}$. (b) Wide-eye stereo pairs of a rotated $90^{\circ}$ view of (a), showing that the model of the IHMs is present only in the slice between the two blue dotted lines in (a), causing the 
neighboring " $h$ " interaction to be far and the "i" and " $\mathrm{j}$ " to be closer to the reader. In a relaxed state, the S2 of the IHM emerges from the top with a slight angle of $6^{\circ}$, causing the helix of IHMs to "float," separated from the backbone surface by about $2 \mathrm{~nm}$. The blocked head is the only part of the IHM that is in contact with the backbone and is covalently connected to it via the S2 and electrostatically connected by three "anchoring" intermolecular interactions: " $h$ " (blocked head SH3 domain) with the extended S2 of an adjacent tail and "i" (blocked head relay/converter) and "j" (blocked head ELC) with the neighboring S2 (see Fig. 7). For a structure color code, see the legend of Fig. 1.

Fig. 4. Wide-eye stereo pairs of a comparison of the blocked head (green) and free head (blue) of the tarantula IHM PDB 3JBH (Figs. 1-2) with the crystal structures of the pre-power stroke closed PDB $1 B R 1^{79}$ (yellow) and transition PDB $1 D L^{16}$ (red). The ELC and RLC were removed to highlight their lever arms, which are in the same plane but have different angles.

Fig. 5. Small angle X-ray solution scattering (SAXS). Integrated scattering intensity (I in arbitrary units) is given as a function of momentum transfer, $q=4 \pi \sin (\theta) / \lambda$, with a scattering angle of $2 \theta$ and a wavelength of $\lambda$. The comparison of model-based (PDB 3DTP and 3JBH) and measured squid HMM scattering profiles ${ }^{15}$ in (a) shows that the models cannot be distinguished based on the scattering data that is currently available. The predicted scattering profiles are based on electron microscopy-derived striated tarantula muscle (PDB 3DTP ${ }^{23}$, PDB 3JBH; Fig. 1) IHM models. Calculated wide-angle scattering data (b) confirms that the models do not significantly differ in the wide angle $\mathrm{X}$-ray solution scattering region.

Fig. 6. Wide-eye stereo pairs showing the general location of intramolecular interactions (a) "a" and "f," (b) "e" and "g," and (c) "d," formed by two sub-interactions, "d.1" and "d.2." (a) and (c) have the same viewpoint as Fig. 2a and (b) has the viewpoint as Fig. 2b.

Fig. 7. Wide-eye stereo pairs of (a) intermolecular interactions "c" and "b" and (b) the anchoring intermolecular interactions " $h$ ", " $i$," and " $j$ ". For clarity, the neighboring S2 has been extended from the IHM S2 as a coiled-coil $\alpha$-helix (pink). The neighboring subfilaments, which have unknown structures, are depicted as cylinders with diameters of about $2.2 \mathrm{~nm}$. (c) Stereo pairs of (b) as viewed transversally from the top, showing interactions " $h$," "i," and "j."

Fig. 8. Sequential formation, disruption, and reformation of intra- and intermolecular interactions ("interactions table") in the tarantula IHM PDB 3JBH model upon relaxation (C), activation (C to $\mathrm{F}$, green arrows) and relaxation after activation ( $\mathrm{F}$ to $\mathrm{C}$, red arrows) according to the cooperative phosphorylation activation $(\mathrm{CPA})$ mechanism $(\mathrm{C}-\mathrm{F})$ proposed for tarantula thick filaments ${ }^{24-27}$ allow explanation of the structural basis of the ATP turnover rates detected in tarantula relaxed and $\mathrm{Ca}^{2+}$-activated states. ${ }^{22}$ (A) Model of a short segment of a precursor tarantula thick filament showing three precursor IHMs with disordered heads and unphosphorylated Ser35 (black circles). The heads do not make any inter- or intramolecular interactions. (B) Only one head of each precursor IHM in the pre-power stroke closed state (Fig. 4, green) can establish the three anchoring intermolecular interactions and dock them as blocked heads (light green) to the backbone. The free head (light blue), also in the pre-power stroke closed conformation (Fig. 4, blue), can establish intramolecular interactions with the docked blocked head, which are needed to assemble the IHM. (C) In a relaxed state, these precursor IHMs become fully functional after half the Ser35 are monophosphorylated by a temporarily activated protein kinase $\mathrm{C}$ (PKC) (brown arrow). PKC can only phosphorylate the fully exposed Ser35 of the free heads (blue heads), as the Ser35 of the blocked heads (green heads) are not accessible. ${ }^{23,}{ }^{24}$ Ser35 monophosphorylation of the free heads allows the free heads to sway away and back by Brownian motion ("swaying" heads) by breaking and reforming the intramolecular interactions (denoted by " \pm " in the "interactions table" and by dotted curved arrows in the illustration). (C-F) The tarantula CPA mechanism proposed for 
tarantula thick filament. ${ }^{24,}{ }^{25}$ The interactions table shows how the intra- and intermolecular interactions established in the relaxed state $(\mathrm{C})$ are progressively removed (denoted by crossing them out) upon activation (D-F), ending in a disordered array (F) with fewer interactions. The table also shows how these interactions are progressively reformed from this final disordered array (F) to a newly achieved ordered relaxed array (C). The IHM model and CPA mechanism allow explanation of the structural origin of the very slow ( $>1800 \mathrm{~s})$, slow $(250-300 \mathrm{~s})$ and fast $(<30 \mathrm{~s})$ ATP turnover rates detected in tarantula striated muscle ${ }^{22}$ in a relaxed state: (1) The very slow rate is associated with unphosphorylated docked blocked heads in the IHM (green heads with motor domains labeled as "VS") and the few unphosphorylated docked free heads in the IHM (not shown); (2) the slow rate is associated with the Ser35 monophosphorylated free heads (blue heads with motor domains labeled as "S"); and (3) the fast rate is associated with the Ser35 monophosphorylated swaying free heads that are undocked from the IHM (blue heads with motor domains labeled as "F"). Therefore, the docked (helically ordered) unphosphorylated blocked heads and the few free heads with a very slow rate, as well as the transiently docked Ser35 monophosphorylated free heads with a slow rate, are responsible for super-relaxation (SRX) in tarantulas, ${ }^{22}$ while the remaining (disordered) swaying free heads that move according to Brownian motion and are undocked from the IHM exhibit the fast rate detected in the disordered relaxed state. ${ }^{22}$ In contrast, the very fast $(<0.1 \mathrm{~s})$ rate detected in the $\mathrm{Ca}^{2+}$-activated state in tarantula striated muscle $^{22}$ is associated with Ser35 monophosphorylated free heads (blue heads with motor domains labeled as "VF"), Ser45 monophosphorylated blocked heads (green heads with motor domains labeled as "VF"), and biphosphorylated free heads (blue heads with motor domains labeled as "VF") that are bound to actin (yellow spheres) on the activated thin filament. FH: free head, BH: blocked head.

References

1. Craig, R. \& Padrón, R. (2004). Molecular structure of the sarcomere. In Myology (Engel, A. G. \& Franzini-Armstrong, C., eds), pp. 129-166, $3^{\text {rd }}$ ed., McGraw-Hill, Inc., New York.

2. Zoghbi, M. E., Woodhead, J. L., Moss, R. L. \& Craig, R. (2008). Three-dimensional structure of vertebrate cardiac muscle myosin filaments. Proc. Natl. Acad. Sci. U. S. A 105, 2386-2390.

3. AL-Khayat, H. A., Kensler, R. W., Squire, J. M., Marston, S. B. \& Morris, E. P. (2013). Atomic model of the human cardiac muscle myosin filament. Proc. Natl. Acad. Sci. U. S. A 110, 318-323.

4. Gonzalez-Sola, M., AL-Khayat, H. A., Behra, M. \& Kensler, R. W. (2014). Zebrafish cardiac muscle thick filaments: isolation technique and three-dimensional structure. Biophys. J. 106, 1671-1680.

5. Woodhead, J. L., Zhao, F. Q., Craig, R., Egelman, E. H., Alamo, L. \& Padrón, R. (2005). Atomic model of a myosin filament in the relaxed state. Nature 436, 1195-1199.

6. Zhao, F. Q., Craig, R. \& Woodhead, J. L. (2009). Head-head interaction characterizes the relaxed state of Limulus muscle myosin filaments. J. Mol. Biol. 385, 423-431.

7. Pinto, A., Sanchez, F., Alamo, L. \& Padron, R. (2012). The myosin interacting-heads motif is present in the relaxed thick filament of the striated muscle of scorpion. J. Struct. Biol. 180, 469-478.

8. Woodhead, J. L., Zhao, F. Q. \& Craig, R. (2013). Structural basis of the relaxed state of a $\mathrm{Ca} 2+-$ regulated myosin filament and its evolutionary implications. Proc. Natl. Acad. Sci. U. S. A 110, 8561-8566.

9. Sulbarán, G., Alamo, L., Pinto, A., Márquez, G., Méndez, F., Padrón, R., and Craig, R. 2014 Schistosome Muscles Contain Striated Muscle-Like Myosin Filaments in a Smooth Muscle-Like Architecture Biophys.J. 106 159a

10. Craig, R. \& Megerman, J. (1977). Assembly of smooth muscle myosin into side-polar filaments. J. Cell Biol. 75, 990-996. 
11. Wendt, T., Taylor, D., Trybus, K. M. \& Taylor, K. (2001). Three-dimensional image reconstruction of dephosphorylated smooth muscle heavy meromyosin reveals asymmetry in the interaction between myosin heads and placement of subfragment 2. Proc. Natl. Acad. Sci. U. S. A 98, 4361-4366.

12. Sulbaran, G., Alamo, L., Pinto, A., Marquez, G., Mendez, F., Padron, R. \& Craig, R. (2015). An invertebrate smooth muscle with striated muscle myosin filaments. Proc. Natl. Acad. Sci. U. S. A 112, E5660-E5668.

13. Jung, H. S., Burgess, S. A., Billington, N., Colegrave, M., Patel, H., Chalovich, J. M., Chantler, P. D. \& Knight, P. J. (2008). Conservation of the regulated structure of folded myosin 2 in species separated by at least 600 million years of independent evolution. Proc. Natl. Acad. Sci. U. S. A 105, 6022-6026.

14. Sulbarán, Mun, J. Y., Lee, K. H., Alamo, L., Pinto, A., Sato, O., Ikebe, M., Liu, X., Korn, E. D., Padrón, R., and Craig, R. 2015 The inhibited, interacting-heads motif characterizes myosin II from the earliest animals with muscle. Biophys.J. 108 301a

15. Gillilan, R. E., Kumar, V. S., O'Neall-Hennessey, E., Cohen, C. \& Brown, J. H. (2013). XRay Solution Scattering of Squid Heavy Meromyosin: Strengthening the Evidence for an Ancient Compact off State. PLoS. One. 8, e81994.

16. Houdusse, A., Szent-Gyorgyi, A. G. \& Cohen, C. (2000). Three conformational states of scallop myosin S1. Proc. Natl. Acad. Sci. U. S. A 97, 11238-11243.

17. Jung, H. S., Komatsu, S., Ikebe, M. \& Craig, R. (2008). Head-head and head-tail interaction: a general mechanism for switching off myosin II activity in cells. Mol. Biol. Cell 19, 3234-3242.

18. Stewart, M. A., Franks-Skiba, K., Chen, S. \& Cooke, R. (2010). Myosin ATP turnover rate is a mechanism involved in thermogenesis in resting skeletal muscle fibers. Proc. Natl. Acad. Sci. U. S. A 107, 430-435.

19. Hooijman, P., Stewart, M. A. \& Cooke, R. (2011). A new state of cardiac myosin with very slow ATP turnover: a potential cardioprotective mechanism in the heart. Biophys. J. 100, 1969-1976.

20. Cooke, R. (2011). The role of the myosin ATPase activity in adaptive thermogenesis by skeletal muscle. Biophys. Rev. 3, 33-45.

21. McNamara, J. W., Li, A., dos Remedios, C. G. \& Cooke, R. (2015). The role of superrelaxed myosin in skeletal and cardiac muscle. Biophysical Reviews 7, 5-14.

22. Naber, N., Cooke, R. \& Pate, E. (2011). Slow Myosin ATP Turnover in the Super-Relaxed State in Tarantula Muscle. J. Mol. Biol.

23. Alamo, L., Wriggers, W., Pinto, A., Bartoli, F., Salazar, L., Zhao, F. Q., Craig, R. \& Padrón, R. (2008). Three-dimensional reconstruction of tarantula myosin filaments suggests how phosphorylation may regulate myosin activity. J. Mol. Biol. 384, 780-797.

24. Brito, R., Alamo, L., Lundberg, U., Guerrero, J. R., Pinto, A., Sulbaran, G., Gawinowicz, M. A., Craig, R. \& Padron, R. (2011). A molecular model of phosphorylation-based activation and potentiation of tarantula muscle thick filaments. J. Mol. Biol. 414, 44-61.

25. Sulbarán, G., Biasutto, A., Alamo, L., Riggs, C., Pinto, A., Mendéz, F., Craig, R. \& Padrón, R. (2013). Different head environments in tarantula thick filaments support a cooperative activation process. Biophys. J. 105, 2114-2122.

26. Espinoza-Fonseca, L. M., Alamo, L., Pinto, A., Thomas, D. D. \& Padron, R. (2015). Sequential myosin phosphorylation activates tarantula thick filament via a disorder-order transition. Mol. Biosyst. 11, 2167-2179.

27. Alamo, L., Li, X. E., Espinoza-Fonseca, L. M., Pinto, A., Thomas, D. D., Lehman, W. \& Padron, R. (2015). Tarantula myosin free head regulatory light chain phosphorylation stiffens N-terminal extension, releasing it and blocking its docking back. Mol. Biosyst. 11, 2180-2189.

28. Fusi, L., Huang, Z. \& Irving, M. (2015). The Conformation of Myosin Heads in Relaxed Skeletal Muscle: Implications for Myosin-Based Regulation. Biophys. J. 109, 783-792. 
29. Woodhead, J. L. \& Craig, R. (2015). Through Thick and Thin-Interfilament Communication in Muscle. Biophys. J. 109, 665-667.

30. Kampourakis, T., Sun, Y. B. \& Irving, M. (2015). Orientation of the N- and C-terminal lobes of the myosin regulatory light chain in cardiac muscle. Biophys. J. 108, 304-314.

31. Kampourakis, T. \& Irving, M. (2015). Phosphorylation of myosin regulatory light chain controls myosin head conformation in cardiac muscle. J. Mol. Cell Cardiol. 85, 199-206.

32. Baumann, B. A., Taylor, D. W., Huang, Z., Tama, F., Fagnant, P. M., Trybus, K. M. \& Taylor, K. A. (2012). Phosphorylated smooth muscle heavy meromyosin shows an open conformation linked to activation. J. Mol. Biol. 415, 274-287.

33. Taylor, K. A., Feig, M., Brooks, C. L., III, Fagnant, P. M., Lowey, S. \& Trybus, K. M. (2014). Role of the essential light chain in the activation of smooth muscle myosin by regulatory light chain phosphorylation. J. Struct. Biol. 185, 375-382.

34. Wendt, T., Taylor, D., Messier, T., Trybus, K. M. \& Taylor, K. A. (1999). Visualization of head-head interactions in the inhibited state of smooth muscle myosin. J. Cell Biol. 147, 1385-1390.

35. Liu, J., Wendt, T., Taylor, D. \& Taylor, K. (2003). Refined model of the $10 \mathrm{~S}$ conformation of smooth muscle myosin by cryo-electron microscopy 3D image reconstruction. J. Mol. Biol. 329, 963-972.

36. Lawson, C. L., Baker, M. L., Best, C., Bi, C., Dougherty, M., Feng, P., van, G. G., Devkota, B., Lagerstedt, I., Ludtke, S. J., Newman, R. H., Oldfield, T. J., Rees, I., Sahni, G., Sala, R., Velankar, S., Warren, J., Westbrook, J. D., Henrick, K., Kleywegt, G. J., Berman, H. M. \& Chiu, W. (2011). EMDataBank.org: unified data resource for CryoEM. Nucleic Acids Res. 39, D456-D464.

37. Blankenfeldt, W., Thoma, N. H., Wray, J. S., Gautel, M. \& Schlichting, I. (2006). Crystal structures of human cardiac beta-myosin II S2-Delta provide insight into the functional role of the S2 subfragment. Proc. Natl. Acad. Sci. U. S. A 103, 17713-17717.

38. Zhu, J., Sun, Y., Zhao, F. Q., Yu, J., Craig, R. \& Hu, S. (2009). Analysis of tarantula skeletal muscle protein sequences and identification of transcriptional isoforms. BMC. Genomics 10, 117.

39. Benson, D. A., Karsch-Mizrachi, I., Lipman, D. J., Ostell, J. \& Wheeler, D. L. (2005). GenBank. Nucleic Acids Res. 33, D34-D38.

40. (2015). UniProt: a hub for protein information. Nucleic Acids Res. 43, D204-D212.

41. Uyeda, T. Q., Ruppel, K. M. \& Spudich, J. A. (1994). Enzymatic activities correlate with chimaeric substitutions at the actin-binding face of myosin. Nature 368, 567-569.

42. Rovner, A. S. (1998). A long, weakly charged actin-binding loop is required for phosphorylation-dependent regulation of smooth muscle myosin. J. Biol. Chem. 273, 27939-27944.

43. Murphy, C. T. \& Spudich, J. A. (2000). Variable surface loops and myosin activity: accessories to a motor. J Muscle Res. Cell Motil. 21, 139-151.

44. Berman, H. M., Bhat, T. N., Bourne, P. E., Feng, Z., Gilliland, G., Weissig, H. \& Westbrook, J. (2000). The Protein Data Bank and the challenge of structural genomics. Nat. Struct. Biol. 7 Suppl, 957-959.

45. Yang, S., Zhao, F. Q., Sulbarán, G., Woodhead, J. L., Alamo, L., Pinto, A., Padrón, R. \& Craig, R. (2015). Improved imaging, 3D reconstruction and homology modelling of tarantula thick filaments. Biophys. J. 589a.

46. Zoghbi, M. E., Woodhead, J. L., Craig, R. \& Padrón, R. (2004). Helical order in tarantula thick filaments requires the "closed" conformation of the myosin head. J. Mol. Biol. 342, 1223-1236.

47. Xu, S., Offer, G., Gu, J., White, H. D. \& Yu, L. C. (2003). Temperature and ligand dependence of conformation and helical order in myosin filaments. Biochemistry 42, 390401. 
48. Rayment, I., Rypniewski, W. R., Schmidt-Base, K., Smith, R., Tomchick, D. R., Benning, M. M., Winkelmann, D. A., Wesenberg, G. \& Holden, H. M. (1993). Three-dimensional structure of myosin subfragment-1: a molecular motor. Science 261, 50-58.

49. O'Neall-Hennessey, E., Reshetnikova, L., Senthil Kumar, V. S., Robinson, H., SzentGyorgyi, A. G. \& Cohen, C. (2013). Purification, crystallization and preliminary X-ray crystallographic analysis of squid heavy meromyosin. Acta Crystallogr. Sect. F. Struct. Biol. Cryst. Commun. 69, 248-252.

50. Yang, S., Woodhead, J. L., Zhao, F. Q., Sulbaran, G. \& Craig, R. (2015). An approach to improve the resolution of helical filaments with a large axial rise and flexible subunits. $J$. Struct. Biol.

51. Miller, M. S., Soto-Adames, F. N., Braddock, J. M., Wang, J. M., Robbins, J., Vigoreaux, J. O. \& Maughan, D. W. (2007). Phylogenic and functional analysis of the myosin light chain amino terminal extensions. J. Muscle Res. Cell Motil. 28, 442.

52. Maughan, D. W., Miller, M. S., Soto-Adames, F. N., Wang, Y., Robbins, J. \& Vigoreaux, J. O. (2008). Phylogenic and functional analysis of the myosin light chain amino terminal extensions. Biophys. J. 94, 306-307.

53. Crowther, R. A., Padrón, R. \& Craig, R. (1985). Arrangement of the heads of myosin in relaxed thick filaments from tarantula muscle. J. Mol. Biol. 184, 429-439.

54. Liu, J., Taylor, D. W., Krementsova, E. B., Trybus, K. M. \& Taylor, K. A. (2006). Threedimensional structure of the myosin $\mathrm{V}$ inhibited state by cryoelectron tomography. Nature 442, 208-211.

55. Geeves, M. A., Fedorov, R. \& Manstein, D. J. (2005). Molecular mechanism of actomyosin-based motility. Cell Mol. Life Sci. 62, 1462-1477.

56. Lorenz, M. \& Holmes, K. C. (2010). The actin-myosin interface. Proc. Natl. Acad. Sci. U. S. A 107, 12529-12534.

57. Craig, R. \& Woodhead, J. L. (2006). Structure and function of myosin filaments. Curr. Opin. Struct. Biol. 16, 204-212.

58. Padrón, R. \& Alamo, L. (2004). Review: The use of negative staining and cryo-electron microscopy to understand the molecular mechanism of myosin-linked regulation of striated muscle contraction. Acta Microscopica 13, 14-29.

59. Burgess, S. A., Yu, S., Walker, M. L., Hawkins, R. J., Chalovich, J. M. \& Knight, P. J. (2007). Structures of smooth muscle myosin and heavy meromyosin in the folded, shutdown state. J. Mol. Biol. 372, 1165-1178.

60. Linari, M., Brunello, E., Reconditi, M., Fusi, L., Caremani, M., Narayanan, T., Piazzesi, G., Lombardi, V. \& Irving, M. (2015). Force generation by skeletal muscle is controlled by mechanosensing in myosin filaments. Nature.

61. Trybus, K. M., Freyzon, Y., Faust, L. Z. \& Sweeney, H. L. (1997). Spare the rod, spoil the regulation: necessity for a myosin rod. Proc. Natl. Acad. Sci. U. S. A 94, 48-52.

62. Craig, R., Padrón, R. \& Kendrick-Jones, J. (1987). Structural changes accompanying phosphorylation of tarantula muscle myosin filaments. J. Cell Biol. 105, 1319-1327.

63. Padrón, R., Pante, N., Sosa, H. \& Kendrick-Jones, J. (1991). X-ray diffraction study of the structural changes accompanying phosphorylation of tarantula muscle. J. Muscle Res. Cell Motil. 12, 235-241.

64. Ravaux, J., Hassanin, A., Deutsch, J., Gaill, F. \& Markmann-Mulisch, U. (2001). Sequence analysis of the myosin regulatory light chain gene of the vestimentiferan Riftia pachyptila. Gene 263, 141-149.

65. Wilson, C., Naber, N., Pate, E. \& Cooke, R. (2014). The Myosin inhibitor blebbistatin stabilizes the super-relaxed state in skeletal muscle. Biophys. J 107, 1637-1646.

66. Colson, B. A., Petersen, K. J., Collins, B. C., Lowe, D. A. \& Thomas, D. D. (2015). The myosin super-relaxed state is disrupted by estradiol deficiency. Biochem. Biophys. Res. Commun. 456, 151-155. 
67. Straight, A. F., Cheung, A., Limouze, J., Chen, I., Westwood, N. J., Sellers, J. R. \& Mitchison, T. J. (2003). Dissecting temporal and spatial control of cytokinesis with a myosin II Inhibitor. Science 299, 1743-1747.

68. Zhao, F. Q., Padron, R. \& Craig, R. (2008). Blebbistatin stabilizes the helical order of myosin filaments by promoting the switch 2 closed state. Biophys. J. 95, 3322-3329.

69. Pettersen, E. F., Goddard, T. D., Huang, C. C., Couch, G. S., Greenblatt, D. M., Meng, E. C. \& Ferrin, T. E. (2004). UCSF Chimera--a visualization system for exploratory research and analysis. J. Comput. Chem. 25, 1605-1612.

70. Humphrey, W., Dalke, A. \& Schulten, K. (1996). VMD: visual molecular dynamics. J. Mol. Graph. 14, 33-38.

71. Kale, L., Skeel, R., Bhandarkar, M., Brunner, R., Gursoy, A., Krawetz, N., Phillips, J., Shinozaki, A. \& Varadarajan, K. S. K. (1999). NAMD2: Greater Scalability for Parallel Molecular Dynamics. J. Comput. Phys. 151, 283-312.

72. Brünger, A. T. (1992). X-PLOR Version 3.1. A system for X-ray Crystallography and NMR Yale University Press.

73. Zhang, L. \& Hermans, J. (1996). Hydrophilicity of cavities in proteins. Proteins 24, 433438.

74. Wriggers, W. \& Chacon, P. (2001). Modeling tricks and fitting techniques for multiresolution structures. Structure. 9, 779-788.

75. Waterhouse, A. M., Procter, J. B., Martin, D. M., Clamp, M. \& Barton, G. J. (2009). Jalview Version 2--a multiple sequence alignment editor and analysis workbench. Bioinformatics. 25, 1189-1191.

76. Larkin, M. A., Blackshields, G., Brown, N. P., Chenna, R., McGettigan, P. A., McWilliam, H., Valentin, F., Wallace, I. M., Wilm, A., Lopez, R., Thompson, J. D., Gibson, T. J. \& Higgins, D. G. (2007). Clustal W and Clustal X version 2.0. Bioinformatics. 23, 2947-2948.

77. Schneidman-Duhovny, D., Hammel, M., Tainer, J. A. \& Sali, A. (2013). Accurate SAXS profile computation and its assessment by contrast variation experiments. Biophys. J. 105, 962-974.

78. Svergun, D., Barberato, C., \& Koch, M. H. J. (1995). CRYSOL - A program to evaluate Xray solution scattering of biological macromolecules from atomic coordinates. J. Appl. Cryst., 28, 768-773.

79. Dominguez, R., Freyzon, Y., Trybus, K. M. \& Cohen, C. (1998). Crystal structure of a vertebrate smooth muscle myosin motor domain and its complex with the essential light chain: visualization of the pre-power stroke state. Cell 94, 559-571. 
Table 1

Conservation of MHC residues involved on critical intramolecular interactions is associated with IHM and SRX presence.

\begin{tabular}{|c|c|c|c|c|c|c|c|c|c|c|c|c|c|c|c|c|c|c|c|c|}
\hline & & Species & $\begin{array}{c}\text { Muscle } \\
\text { type }\end{array}$ & $\begin{array}{c}\text { Filament } \\
\text { type }\end{array}$ & $\begin{array}{l}\text { MHC } \\
\text { type }\end{array}$ & $\begin{array}{c}\text { Myosin } \\
\text { entry }\end{array}$ & $\begin{array}{c}\mathrm{d} .1 \\
\mathrm{i}\end{array}$ & $\begin{array}{c}\text { d.1 } \\
\text { ii }\end{array}$ & $\begin{array}{c}\text { d.1 } \\
\text { iii }\end{array}$ & $\begin{array}{c}\mathrm{d} .2 \\
\mathrm{i}\end{array}$ & $\begin{array}{c}\mathrm{d} .2 \\
\mathrm{ii}\end{array}$ & $\begin{array}{c}\mathrm{d} .2 \\
\mathrm{iii}\end{array}$ & $\begin{array}{c}f .2 \\
i\end{array}$ & $\begin{array}{c}\mathrm{f} .2 \\
\mathrm{ii}\end{array}$ & $\begin{array}{l}\mathrm{f} .2 \\
\mathrm{iii} \\
\end{array}$ & $\begin{array}{c}C_{s-d} \\
\%\end{array}$ & $\begin{array}{l}\mathrm{C}_{\mathrm{s}-\mathrm{f}} \\
\%\end{array}$ & $\begin{array}{l}C_{s} \\
\%\end{array}$ & $\begin{array}{c}\text { IHM } \\
\text { detected? }\end{array}$ & $\begin{array}{c}\text { SRX } \\
\text { detected? }\end{array}$ \\
\hline \multirow{11}{*}{ 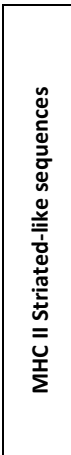 } & \multirow{7}{*}{ 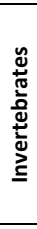 } & $\mathrm{CON}$ & $\mathrm{Sm}$ & Bipolar & St & (a) & 100 & 100 & 100 & $\mathrm{n} / \mathrm{a}$ & 100 & $\mathrm{n} / \mathrm{a}$ & 50 & 100 & 100 & $\mathrm{n} / \mathrm{a}$ & 83 & $\mathrm{n} / \mathrm{a}$ & yes & - \\
\hline & & $\mathrm{APH}$ & St & Bipolar & St & (b) & 100 & 100 & 100 & 100 & 100 & 100 & 100 & 100 & 100 & 100 & 100 & 100 & yes & yes \\
\hline & & DROe & St & Bipolar & St & P05661 & 100 & 0 & 100 & 66.7 & 100 & 83.3 & 62.5 & 100 & 100 & 75 & 88 & 79 & - & - \\
\hline & & DRO & St & Bipolar & St & P05661 & 100 & 0 & 100 & 0 & 100 & 83.3 & 62.5 & 100 & 100 & 64 & 88 & 72 & - & - \\
\hline & & $\mathrm{SCH}$ & $\mathrm{Sm}$ & Bipolar & St & Q02456 & 100 & 100 & 100 & 0 & 100 & 100 & 62.5 & 100 & 100 & 83 & 88 & 85 & yes & - \\
\hline & & DOR & St & $?$ & St & G4V4Y6 & 100 & 100 & 100 & 0 & 100 & 100 & 56.3 & 100 & 88 & 83 & 81 & 83 & yes (c) & - \\
\hline & & PLA & St & Bipolar & St & Q26079 & 100 & 100 & 100 & 0 & 100 & 100 & 56.3 & 100 & 100 & 83 & 85 & 84 & yes & - \\
\hline & \multirow{3}{*}{ 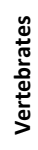 } & MUS & $\mathrm{Ca}$ & Bipolar & St & Q91Z83 & 100 & 100 & 92 & 0 & 100 & 100 & 87.5 & 100 & 88 & 82 & 92 & 85 & yes & - \\
\hline & & HOM & $\mathrm{Ca}$ & Bipolar & St & P12883 & 100 & 100 & 92 & 0 & 100 & 100 & 87.5 & 100 & 88 & 82 & 92 & 85 & yes & yes (d) \\
\hline & & DAN & $\mathrm{Ca}$ & Bipolar & St & Q9PVE1 & 100 & 100 & 92 & 0 & 75 & 100 & 87.5 & 100 & 88 & 78 & 92 & 82 & yes & - \\
\hline & $\underline{\dot{z}}$ & AMP & St & No & St & I1GH71 & 100 & 100 & 66.7 & 0 & 75 & 75 & 62.5 & 100 & 100 & 69 & 87 & 75 & - & - \\
\hline \multirow{5}{*}{ 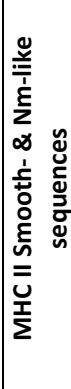 } & \multirow{2}{*}{ 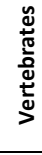 } & GAL & $\mathrm{Sm}$ & Side-polar & $\mathrm{Sm}$ & P10587 & 100 & 100 & 92 & 92 & 75 & 50 & 87.5 & 100 & 96 & 85 & 94 & 88 & yes & yes (e) \\
\hline & & MUS & $\mathrm{Nm}$ & $\begin{array}{c}\text { Bipolar } \\
\text { Mini- } \\
\text { filamets }\end{array}$ & $\mathrm{Nm}$ & Q61879 & 100 & 100 & 92 & 92 & 75 & 50 & 87.5 & 100 & 96 & 85 & 94 & 88 & yes & - \\
\hline & $\underline{\underline{I}}$ & AMP & $\mathrm{Nm}$ & No & $\mathrm{Nm}$ & 1GH58 & 100 & 100 & 0 & 0 & 66.7 & 50 & 68.8 & 100 & 79.2 & 53 & 83 & 63 & - & - \\
\hline & \multirow{2}{*}{ 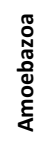 } & DIC & $\mathrm{Nm}$ & $\begin{array}{c}\text { Mini- } \\
\text { filaments }\end{array}$ & $\mathrm{Nm}$ & P08799 & 75 & 0 & 66.7 & 0 & 0 & 0 & 0 & 58.3 & 62.5 & 24 & 40 & 29 & - & - \\
\hline & & ACA & $\mathrm{Nm}$ & $\begin{array}{c}\text { Mini- } \\
\text { filaments }\end{array}$ & $\mathrm{Nm}$ & P05659 & 0 & 0 & 100 & 0 & 0 & 66.7 & 68.8 & 0 & 50 & 28 & 40 & 32 & no & - \\
\hline \multirow{3}{*}{ 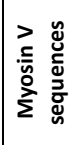 } & \multirow{3}{*}{ 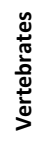 } & Va & - & - & - & Q9Y4I1 & 88 & 0 & 92 & 0 & 75 & 50 & 0 & 0 & 92 & 51 & 31 & 44 & no & - \\
\hline & & $\mathrm{Vb}$ & - & - & - & Q9ULV0 & 63 & 0 & 92 & 0 & 75 & 41.7 & 0 & 0 & 79.2 & 45 & 26 & 39 & no & - \\
\hline & & Vc & - & - & - & Q9NQX4 & 0 & 0 & 66.7 & 0 & 75 & 41.7 & 0 & 0 & 88 & 31 & 29 & 30 & no & - \\
\hline
\end{tabular}

The conservation of positive and negative residues required for establishing each one of the critical "d.1", "d.2" and "f.2", intra-molecular interactions in a specific species vs. tarantula (Aphonopelma) are shown as a percentage (\%) as well as the conservation score $\mathrm{C}_{\mathrm{s}}(\%)$ integrating these interactions altogether (see Material and Methods). The score for the contributions of "d.1" and "d.2" $\left(\mathrm{C}_{\mathrm{s}-\mathrm{d}}\right)$ related to IHM stability and "f.2" ( $\left.\mathrm{C}_{\mathrm{s}-\mathrm{f}}\right)$ related to IHM formation are also shown. The amino acids involved on both sides of the interactions analyzed were: "d.1" i: D391 R164, ii: K394 K400 E165, iii: E406 K449 R659; "d.2" i: E292 D302 K734, ii: E371 K763 R500, iii: E376 E379 K721 and "f.2" i: E893 E895 R659 H665, ii: D901 E904 K523, iii: R905 K908 K913 E532 E533 E534. Acronyms used for the species are: CON: Condylactis gigantea (anemone), APH: Aphonopelma sp. (tarantula), DROe: Drosophila melanogaster (flight muscle embryonic isoform), DRO: Drosophila melanogaster (flight muscle), SCH: Schistosome mansoni, DOR: Doryteuthis pealeii (squid), PLA: Placopecten magellanicus (scallop), MUS: Mus musculus (mouse), HOM: Homo sapiens, DAN: Danio rerio (zebrafish), AMP: Amphimedon (sponge), GAL: Gallus (chicken), ACA: Acanthamoeba castellani, DIC: Dictyostelium discoideum, Va-c: myosin V Homo sapiens isoforms. The MHC entry sequences are detailed in Suppl. Tables 3. Muscle and MHC types: St: Striated, Ca: Cardiac, Sm: Smooth, Nm: Nonmuscle. Notes: (a) For anemone only partial MHC sequences (Aulactinia verrucosa Q8MV94, Anemonia sulcata Q8MV95, Nematostella vectensis I2G9D5) were available for the interactions as follows: "d.1" i (Nematostella), ii (Nematostella), iii (Nematostella, Aulactinia, Anemonia), "d.2"ii (Nematostella, Aulactinia) and "f.2" i-iii (Aulactinia, Anemonia). n/a represents lack of sequence information in two anemone MHC sequences. (b) Tarantula Aphonopelma MHC sequence (KT619079) is shown in Supplementary Data. (c) IHM presence was concluded by comparing the SAXS profile of Loligo peali (squid) with the predicted scattering profile for tarantula PDB 3DTP ${ }^{13}$. (d) The presence of SRX state has been confirmed in human cardiac muscle (Dr. James W. McNamara and Roger Cooke, personal communication) and Oryctolagus (rabbit) cardiac muscle whose MHC sequence is very similar to the mouse one so it was not included in Suppl. Table 3. (e) The presence of SRX state has been confirmed in chicken gizzard smooth muscle (preliminary result, Dr. Ed Pate and Dr. Roger Cooke, personal communication). 
a

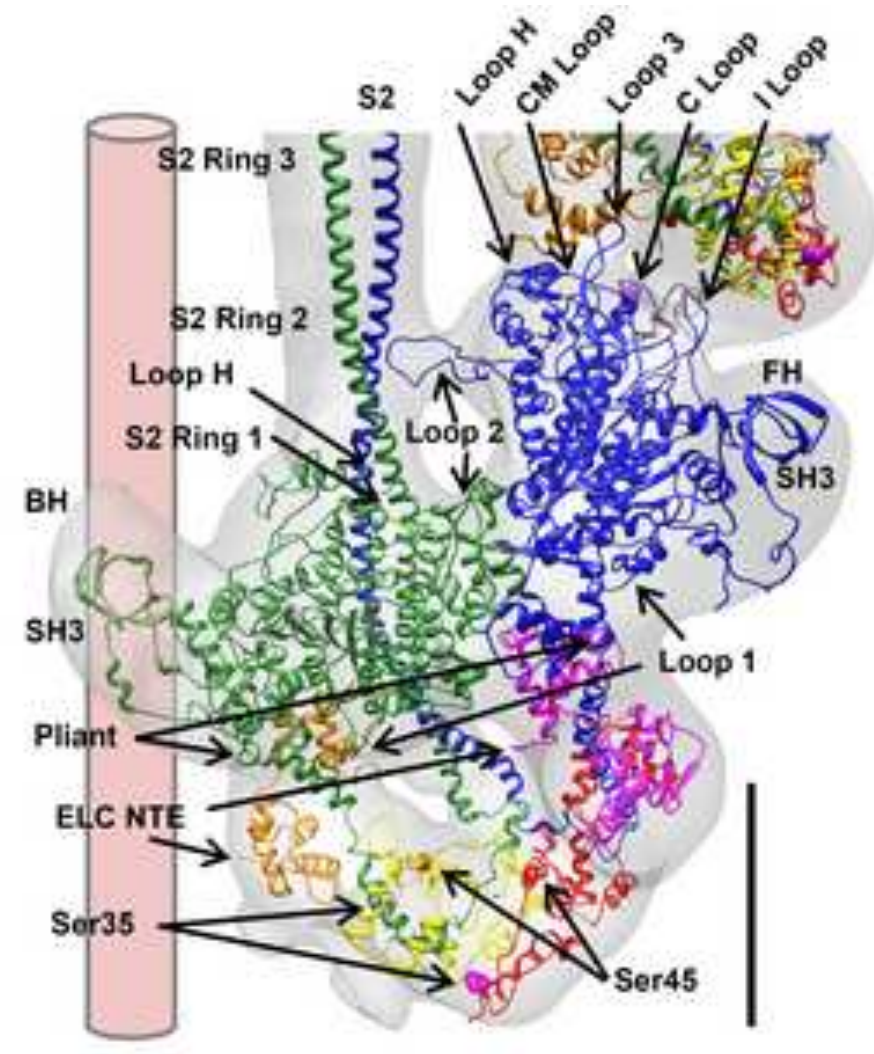

b

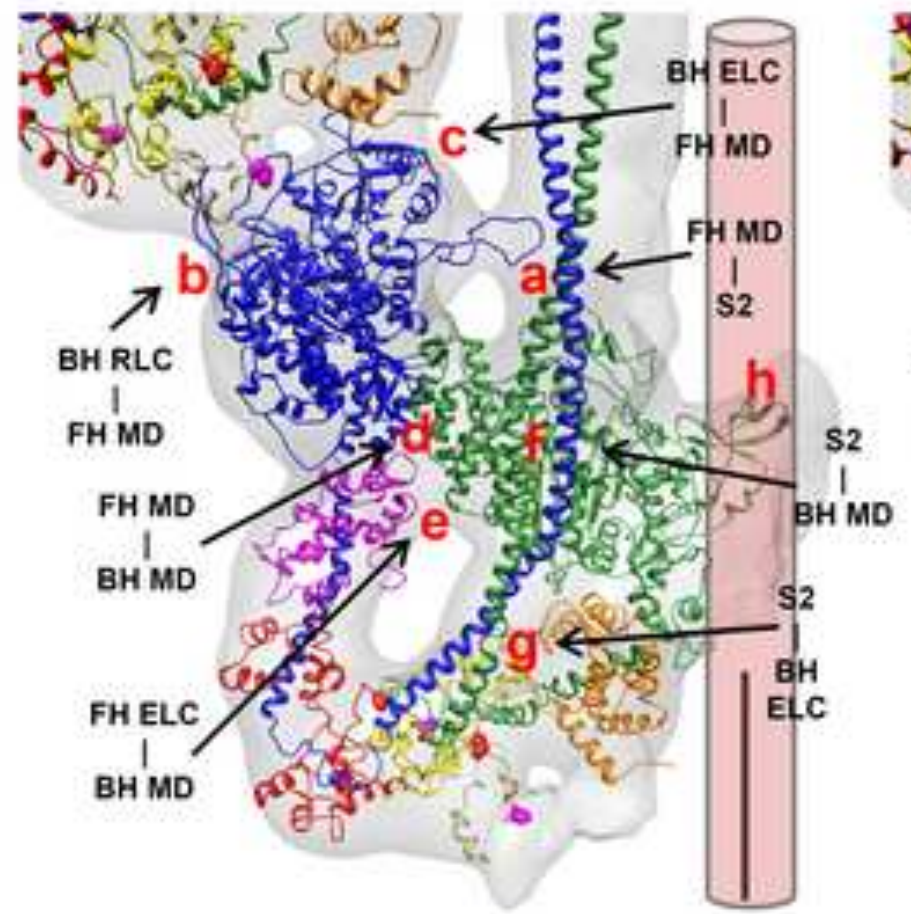

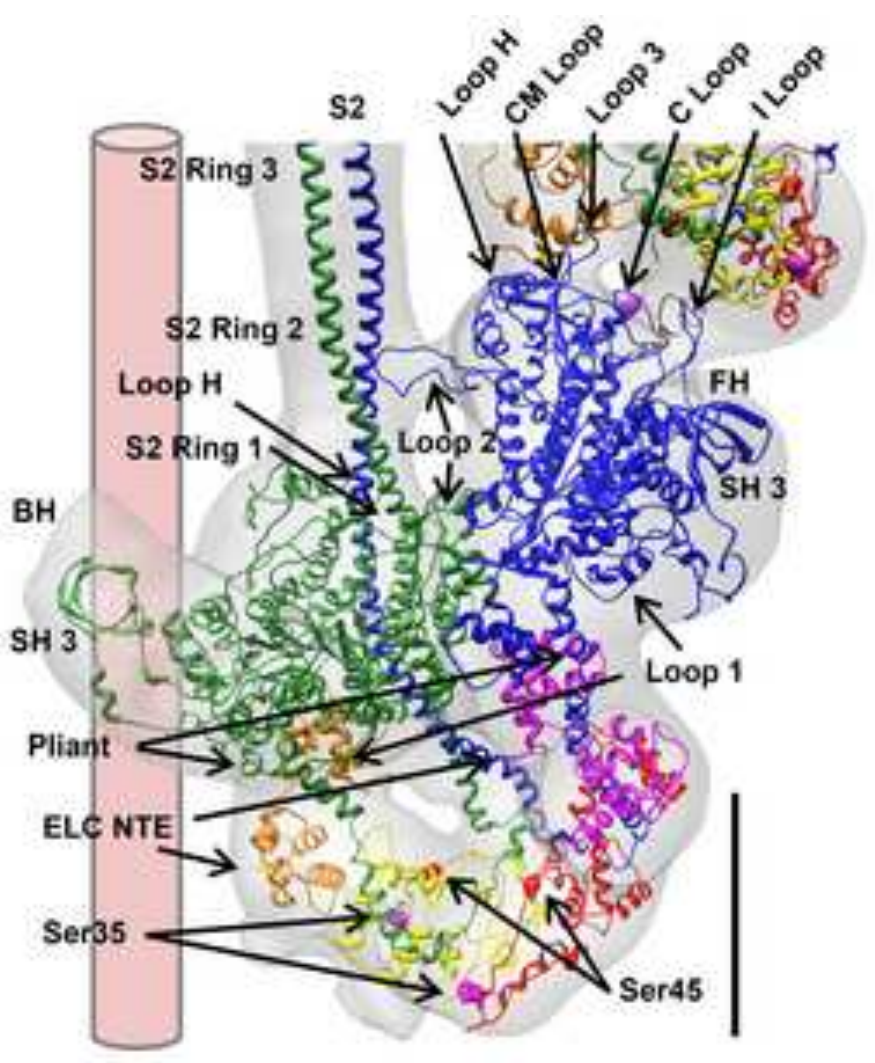

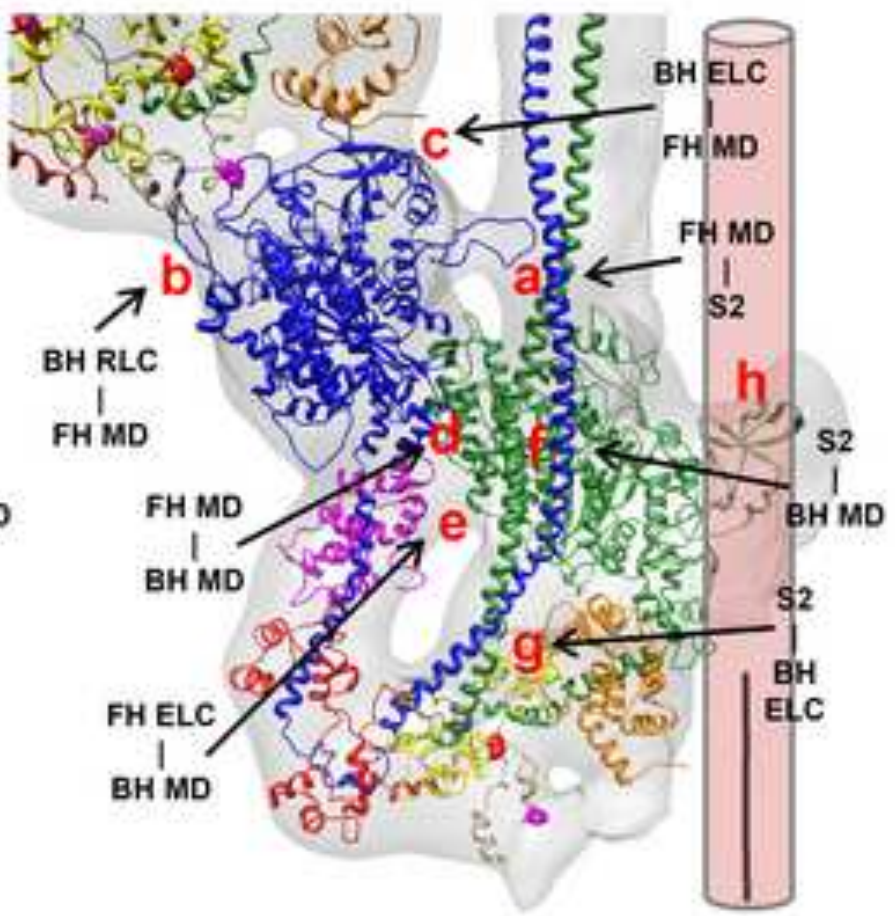


a

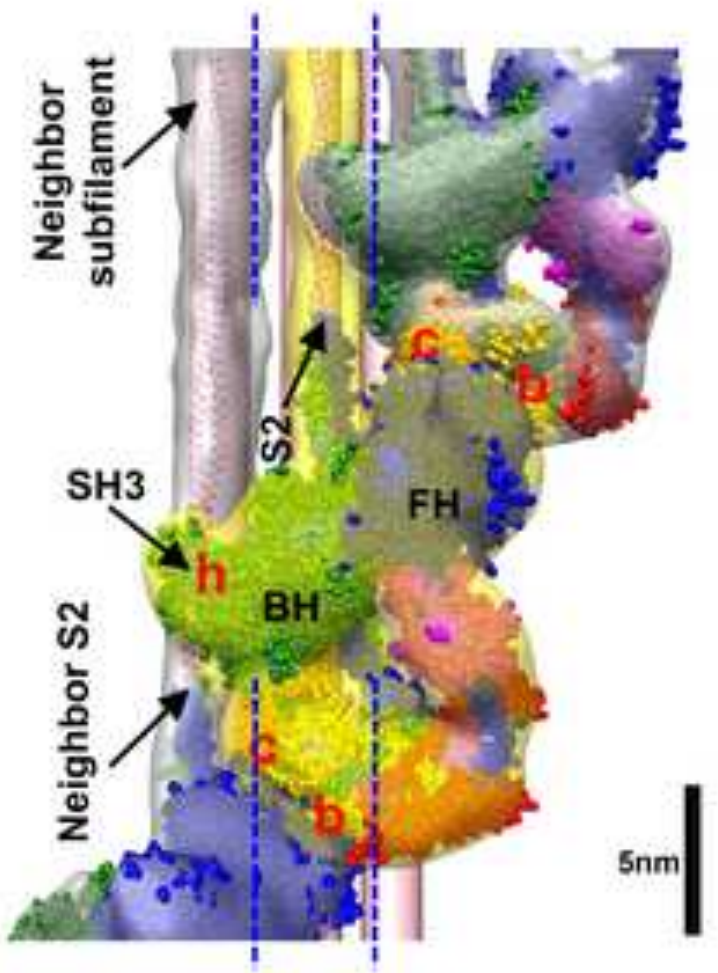

b

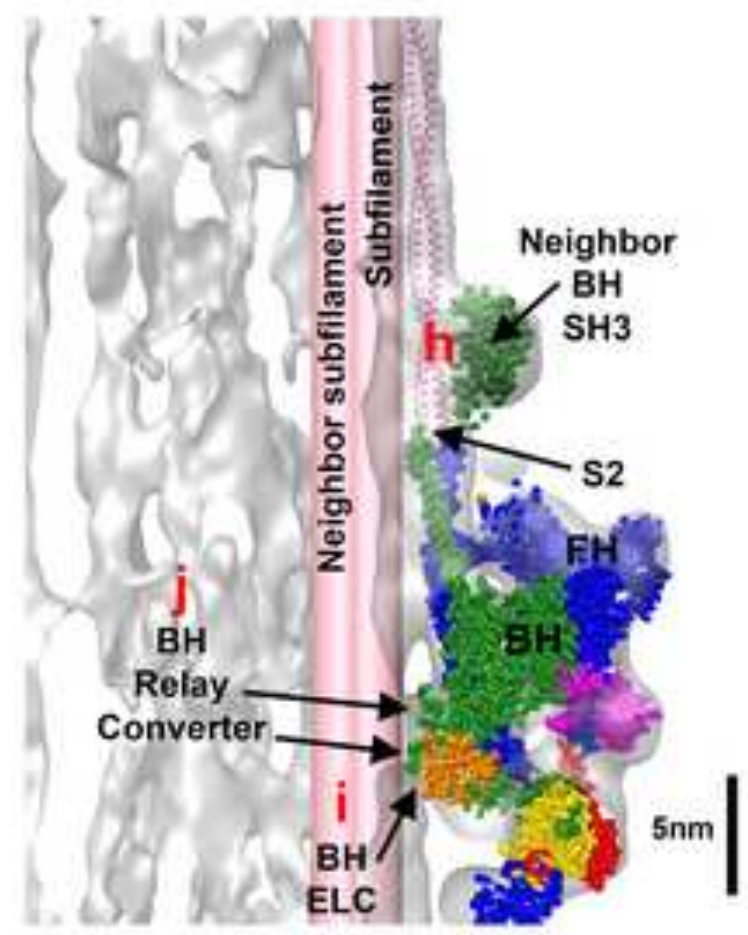

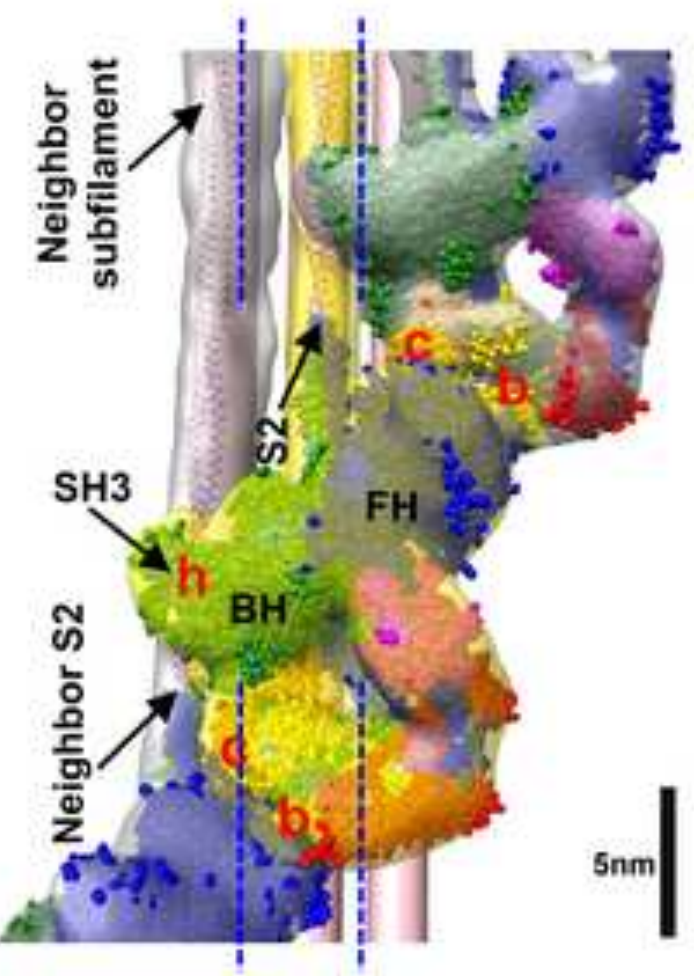

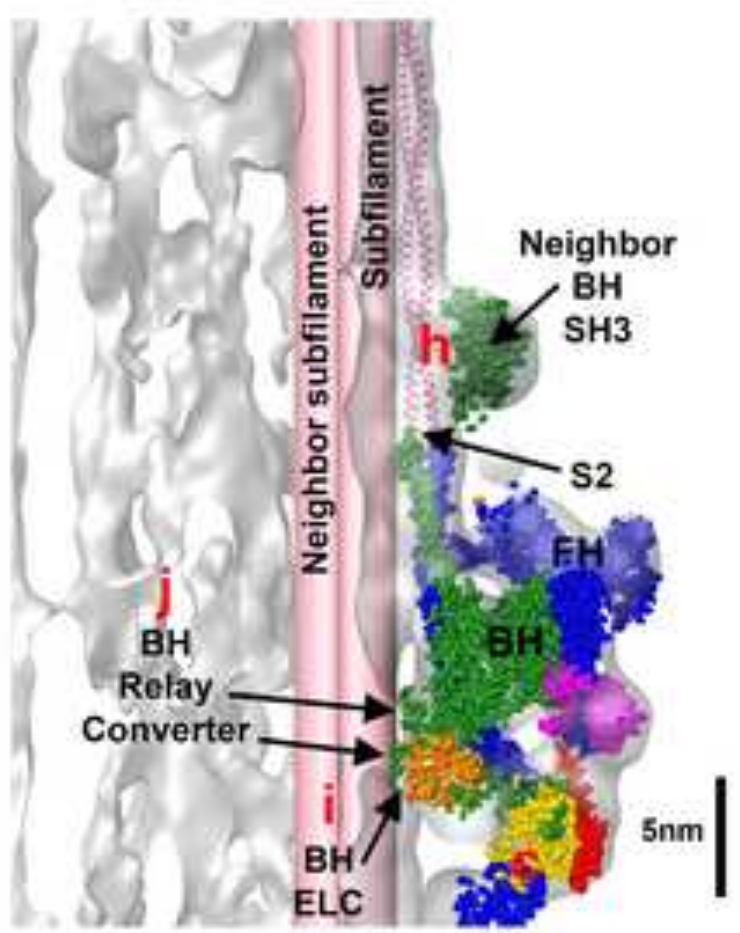



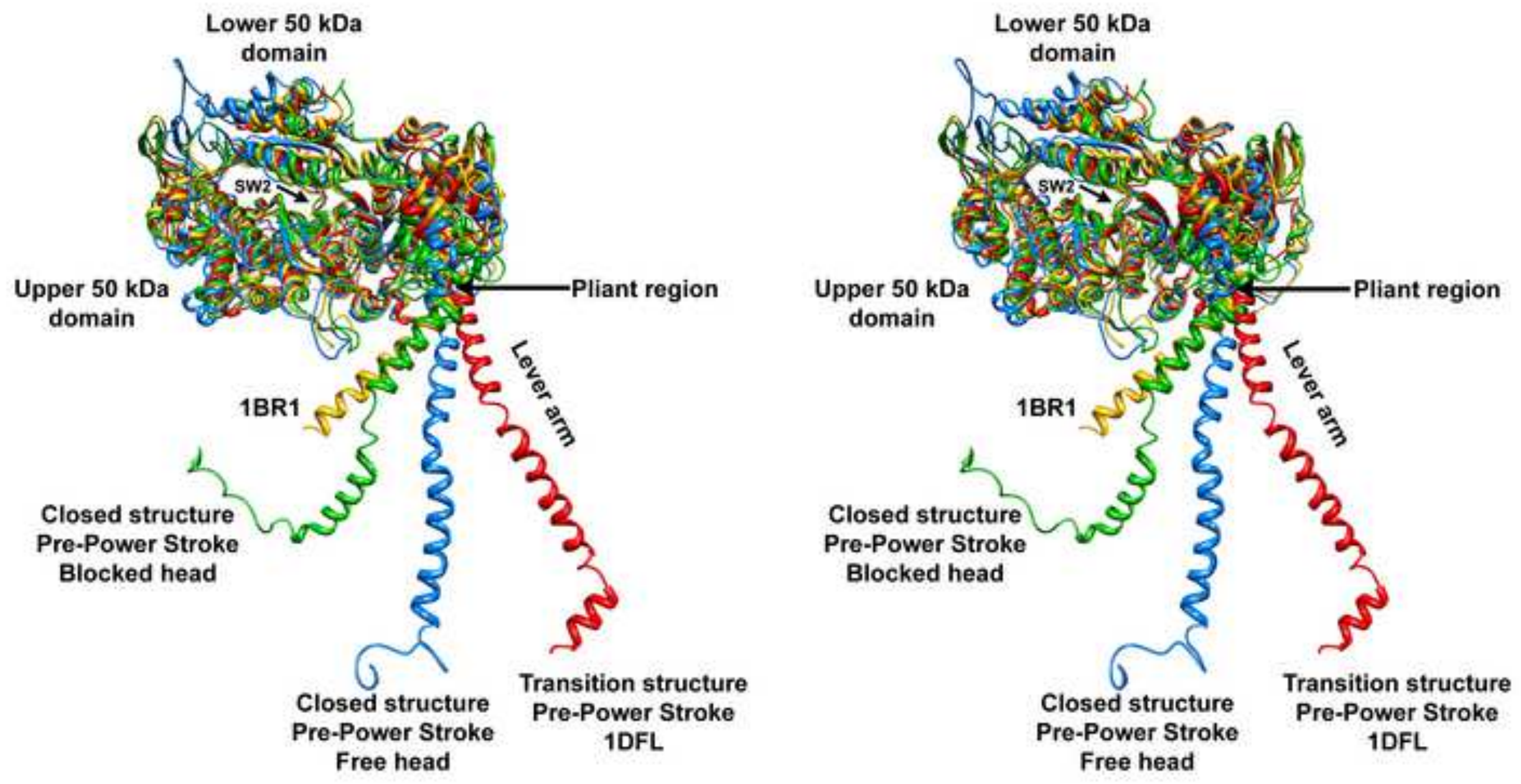
Figure 5
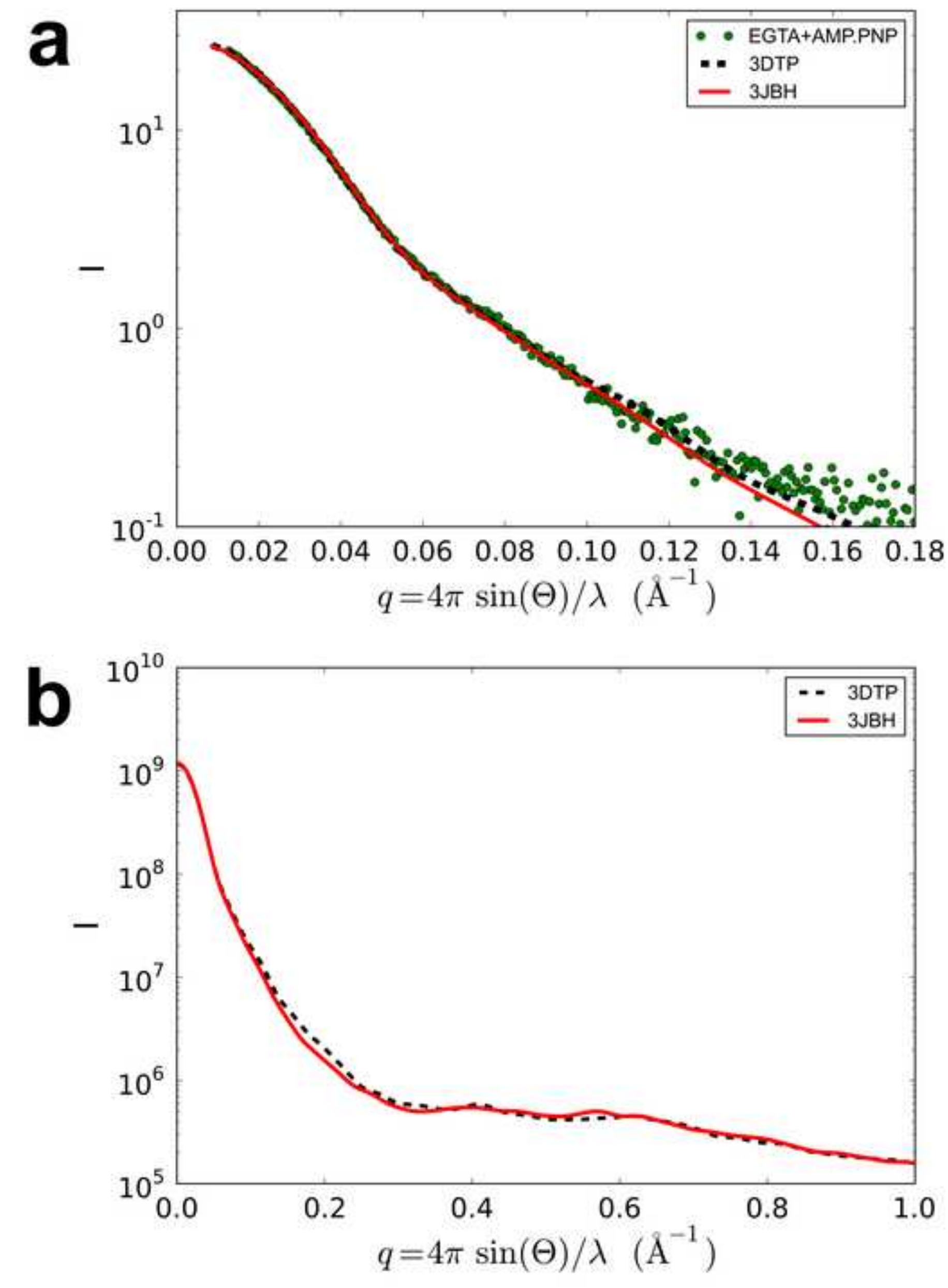

$$
q=4 \pi \sin (\Theta) / \lambda\left(\AA^{-1}\right)
$$


a
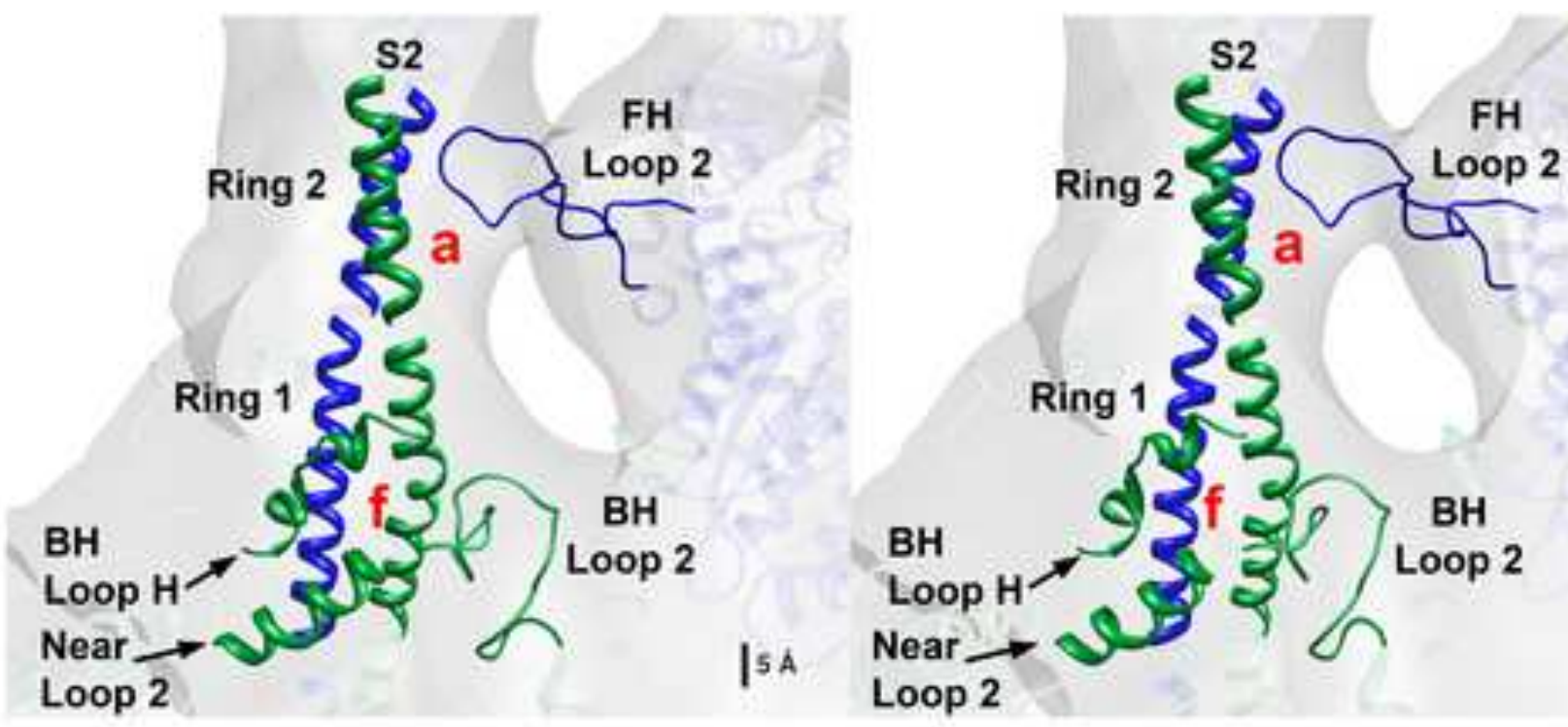

I5 A

b
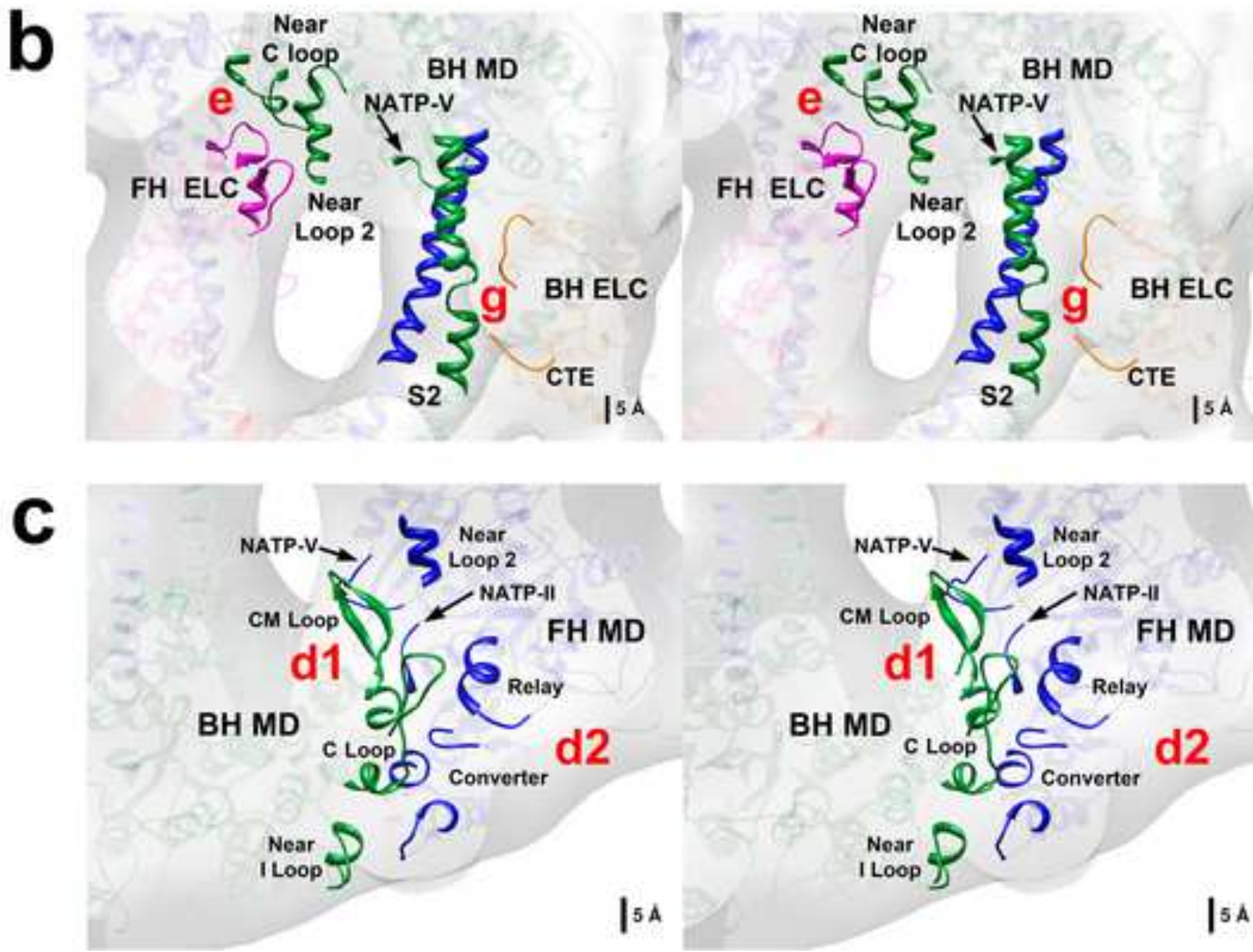


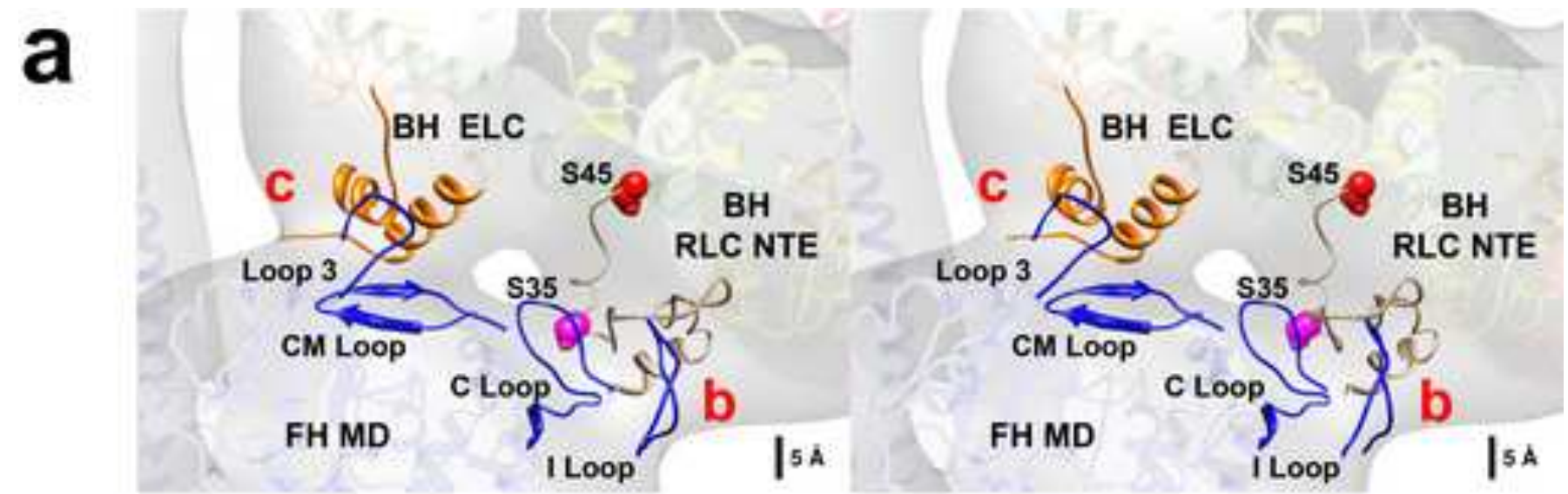

\section{b}
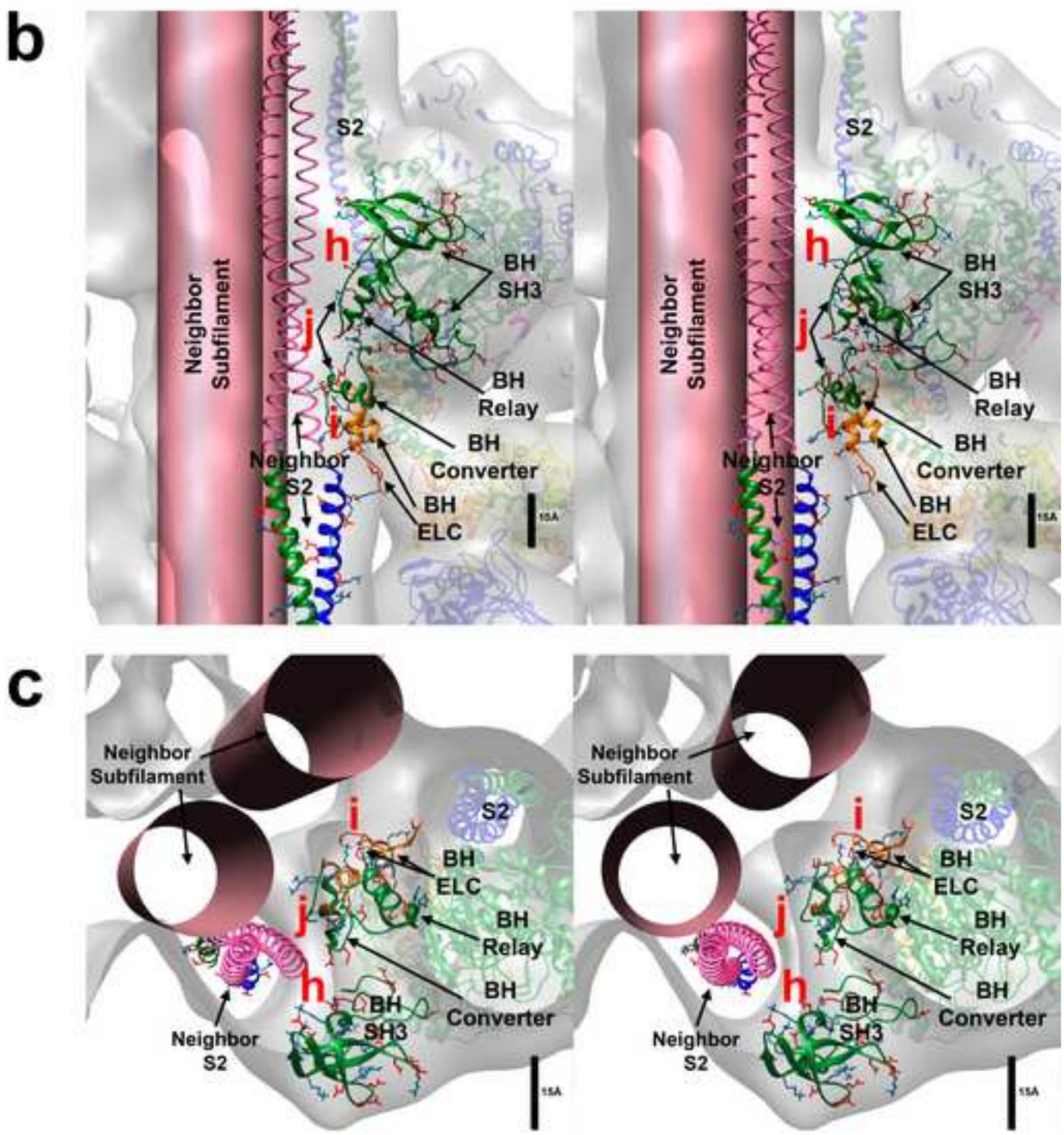


\begin{tabular}{|c|c|c|c|c|c|c|}
\hline \multicolumn{3}{|c|}{ Interactions table } & $\begin{array}{c}\text { Relaxed } \\
\text { state }\end{array}$ & $\underset{-\mathrm{Ca}^{2+}+\mathrm{MLCP}}{\stackrel{\mathrm{Ca}^{2+}}{4} \text {.CaM.MLCK }}$ & $\begin{array}{c}\text { Activated } \\
\text { state }\end{array}$ & \\
\hline Intra. & none & $B H s=g, f$ & $\begin{array}{l}\text { Top BH: }, f_{f} \\
\text { Middle BH: } g, f \\
\text { Bottom BH: } \mathrm{g} . \mathrm{f}\end{array}$ & $\begin{array}{l}\text { Top BH: }, \mathrm{f} f \\
\text { Middle } \mathrm{BH}: \mathrm{g}, \mathrm{f} \\
\text { Bottom } \mathrm{BH}: \mathrm{g}, \mathrm{f}\end{array}$ & $\begin{array}{l}\text { Top BH: } \pm \mathrm{g}, \mathrm{f} \\
\text { Middle BH: } \mathrm{g}, \mathrm{f} \\
\text { Bottom } 8 \mathrm{H}: \mathrm{g}, \mathrm{f}\end{array}$ & $\begin{array}{l}\text { Top BH: } \mathrm{g}_{\mathrm{f}} \mathrm{f} \\
\text { Middle } \mathrm{BH}: \mathrm{grf} \\
\text { Bottom BH: } \mathrm{g}, \mathrm{f}\end{array}$ \\
\hline Intra- & none & FHs: none & $\begin{array}{l}\text { Top FH: } \pm \text { RLC-RLC, e,d,a } \\
\text { Mid FH: } \pm \text { RLC-RLC, e,d,a } \\
\text { Bott FH: } \pm \text { RLC-RLC, e,d,a }\end{array}$ & $\begin{array}{l}\text { Top FH: }: \pm \text { RLC-RLC,e,d,a } \\
\text { Mid FH: RLC-RLC, e,d,a } \\
\text { Bott FH: } \pm \text { RLC-RLC, e,d,a }\end{array}$ & $\begin{array}{l}\text { Top FH: } \pm \text { RLC-RLC, e,d,a } \\
\text { Mid FH:RLC-RLC,e,d,a } \\
\text { Bott FH: } \pm \text { RLC-RLC, e,d,a }\end{array}$ & $\begin{array}{l}\text { Top FH: a,d,e, RLC-RIG } \\
\text { Mid FH: RLC RtC,e,d,a } \\
\text { Bott FH: } \pm \text { RLC-RLC, e,d,a }\end{array}$ \\
\hline Inter- & none & BHs; $h, j_{2} i$ & $\begin{array}{c}\text { Top BH: h, j, } \\
\text { Middle BH: } h_{, j, i} \\
\text { Bottom } B H: h, j, i\end{array}$ & $\begin{array}{c}\text { Top } B \mathrm{H}: h, j, \mathrm{I} \\
\text { Middle BH: h,j,i } \\
\text { Bottom } 8 \mathrm{H}: h, j, \mathrm{i}\end{array}$ & $\begin{array}{l}\text { Top } B H: \pm h, j, I \\
\text { Middle BH: h, }, j, \\
\text { Bottom BH: h,j, }\end{array}$ & $\begin{array}{c}\text { Top BH: hi,h } \\
\text { Middle BH: h,j,i } \\
\text { Bottom BH: h,j, }\end{array}$ \\
\hline Inter- & none & FHs: none & $\begin{array}{c}\text { Top FH: } \pm b, c \\
\text { Middle FH: } \pm b, c \\
\text { Bottom } F H: \pm b, c\end{array}$ & $\begin{array}{c}\text { Top FH: }: b, c \\
\text { Middle FH:b,e } \\
\text { Bottom FH: } \pm b, c\end{array}$ & $\begin{array}{l}\text { Top FH: }: b, c \\
\text { Middle FH:b,e } \\
\text { Bottom } F H: \pm b, c\end{array}$ & $\begin{array}{c}\text { Top FH:b,c } \\
\text { Middle FH: } b, e \\
\text { Bottom FH: } \pm b, c\end{array}$ \\
\hline & 8 & 8 & Biocked 15 & pser4s 8 & & \\
\hline
\end{tabular}

ATP turnover rates: very slow (VS) $=1800 \mathrm{~s}$, slow $(\mathrm{S})=250-300 \mathrm{~s}$, fast $(\mathrm{F})<30 \mathrm{~s}$, very fast $(\mathrm{VF})<0.1 \mathrm{~s}$ 


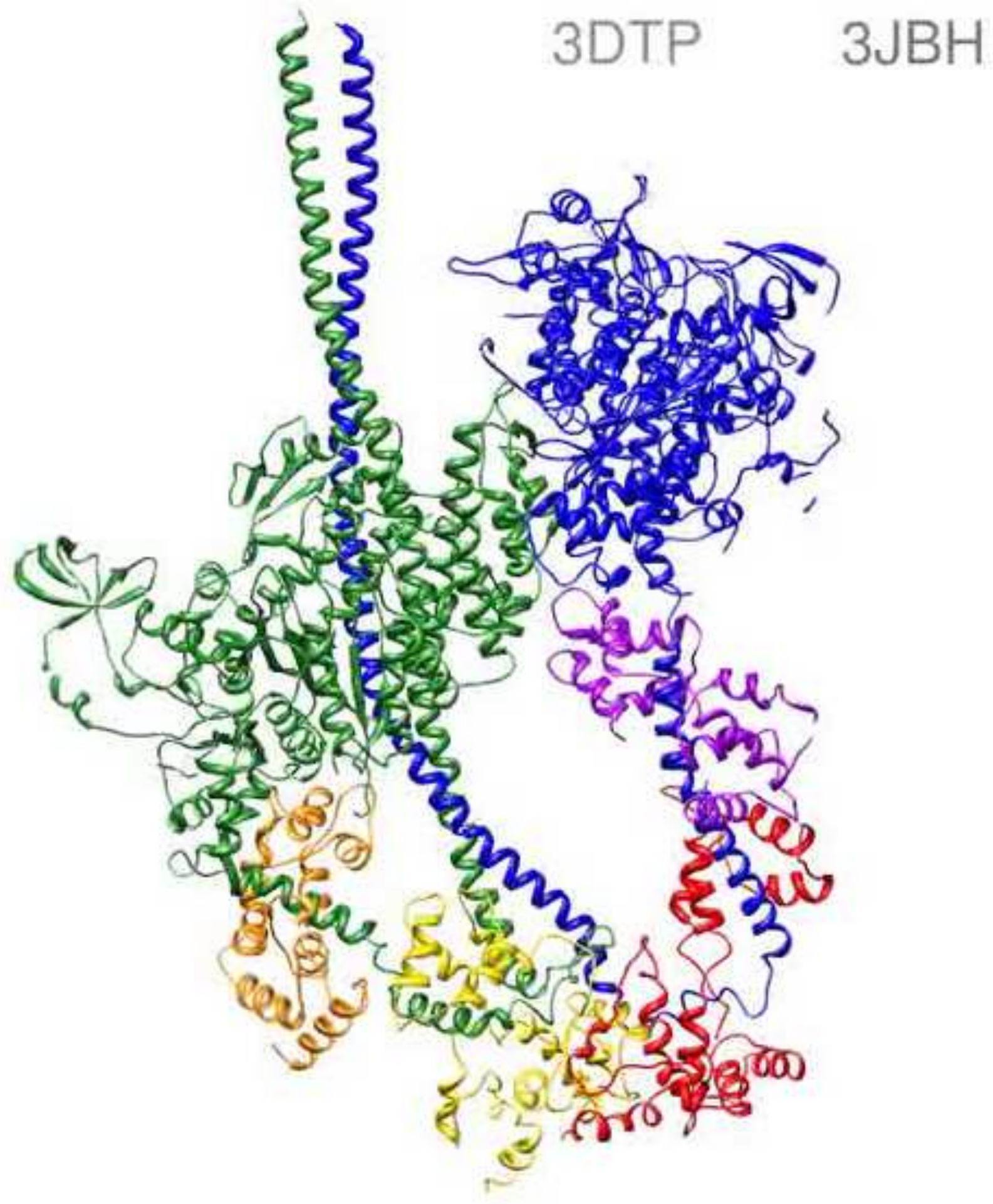

A 


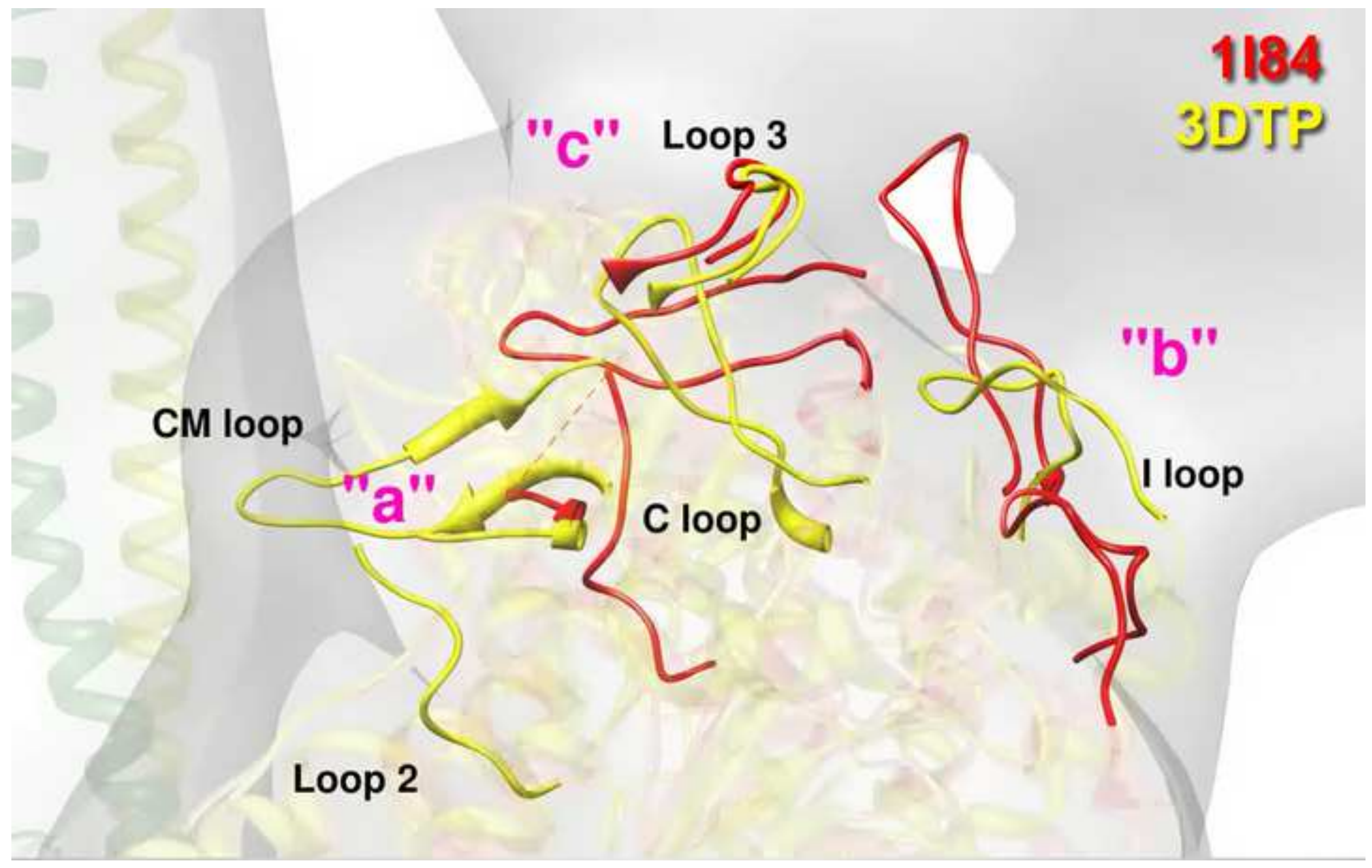




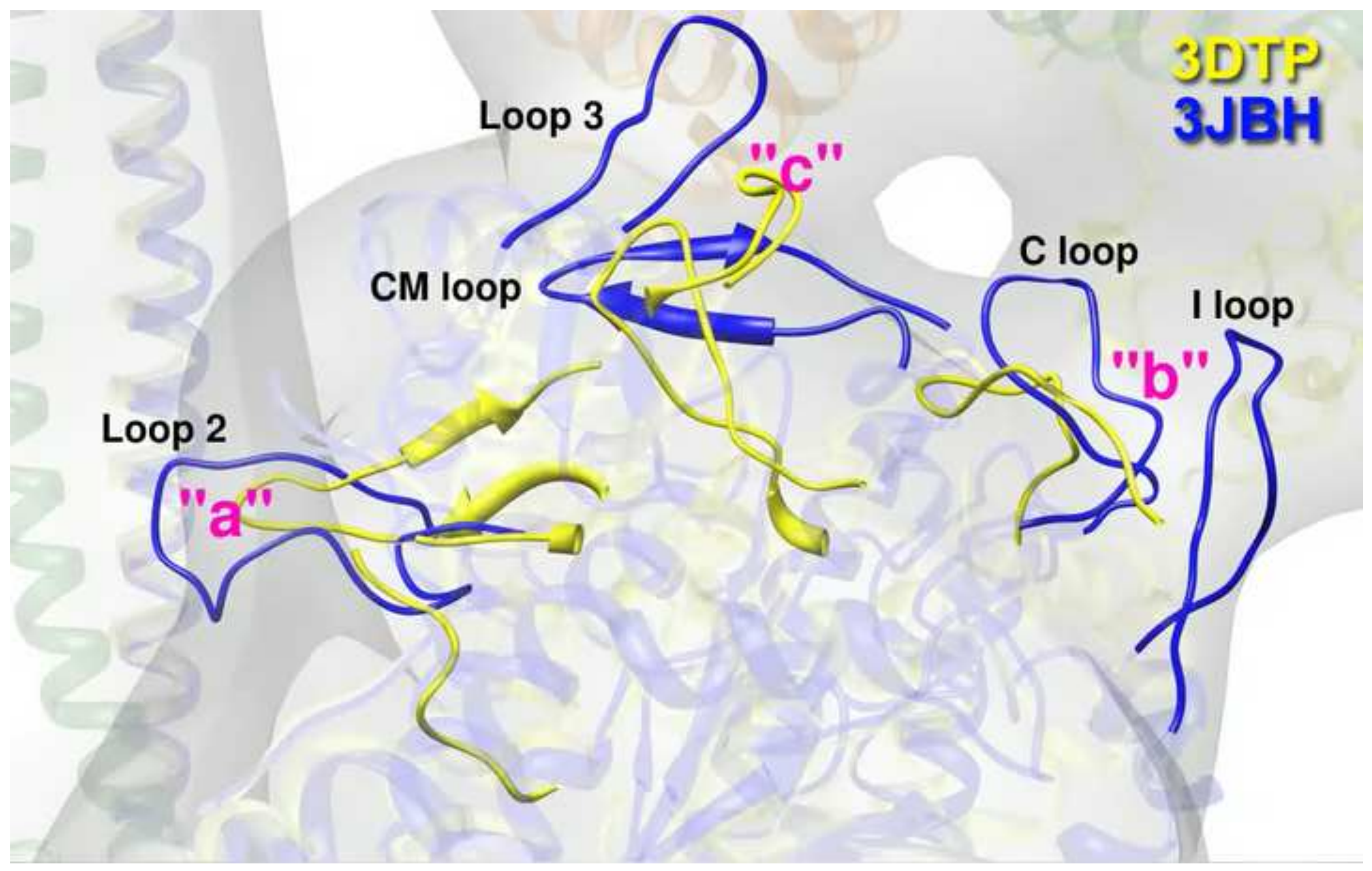




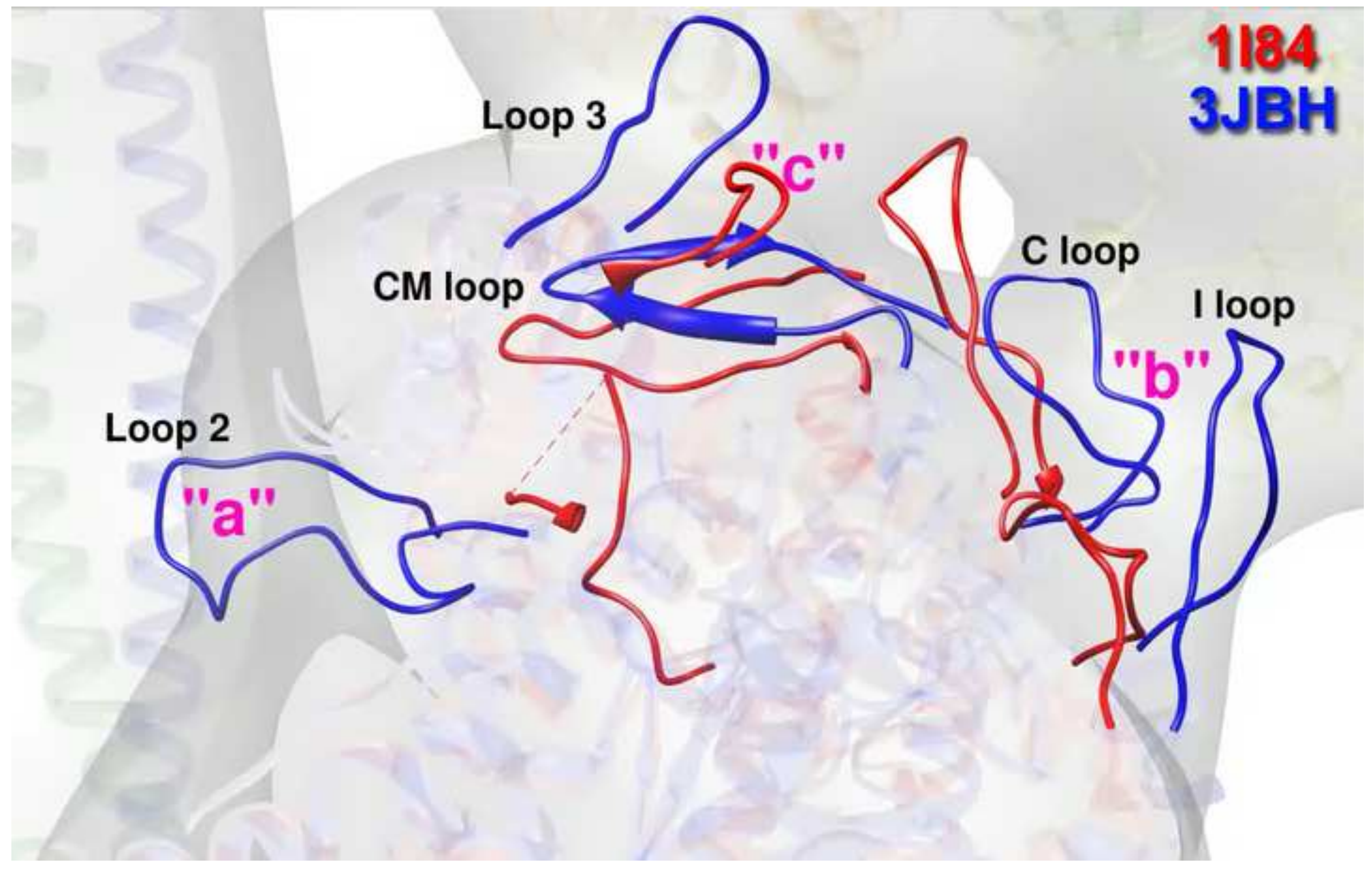

